$12-5-2014$

\title{
Comparing Dense Galaxy Cluster Redshift Surveys with Weak-lensing Maps
}

Ho Seong Hwang

Margaret J. Geller

Antonaldo Diaferio

Kenneth J. Rines

Western Washington University, ken.rines@wwu.edu

J.Jabran Zahid

Follow this and additional works at: https://cedar.wwu.edu/physicsastronomy_facpubs

Part of the Astrophysics and Astronomy Commons

\section{Recommended Citation}

Hwang, Ho Seong; Geller, Margaret J.; Diaferio, Antonaldo; Rines, Kenneth J.; and Zahid, J. Jabran, "Comparing Dense Galaxy Cluster Redshift Surveys with Weak-lensing Maps" (2014). Physics \& Astronomy. 26.

https://cedar.wwu.edu/physicsastronomy_facpubs/26 


\title{
COMPARING DENSE GALAXY CLUSTER REDSHIFT SURVEYS WITH WEAK-LENSING MAPS
}

\author{
Ho Seong Hwang ${ }^{1,2}$, Margaret J. Geller ${ }^{1}$, Antonaldo Diaferio ${ }^{3,4}$, Kenneth J. Rines $^{5}$, and H. Jabran Zahid ${ }^{1}$ \\ ${ }^{1}$ Smithsonian Astrophysical Observatory, 60 Garden Street, Cambridge, MA 02138, USA; \\ hhwang@cfa.harvard.edu,mgeller@cfa.harvard.edu, harus.zahid@cfa.harvard.edu \\ ${ }^{2}$ School of Physics, Korea Institute for Advanced Study, 85 Hoegiro, Dongdaemun-Gu, 130-722 Seoul, Korea \\ ${ }^{3}$ Dipartimento di Fisica, Università degli Studi di Torino, V. Pietro Giuria 1, I-10125 Torino, Italy; diaferio@ @h.unito.it \\ ${ }^{4}$ Istituto Nazionale di Fisica Nucleare (INFN), Sezione di Torino, V. Pietro Giuria 1, I-10125 Torino, Italy \\ ${ }^{5}$ Department of Physics and Astronomy, Western Washington University, Bellingham, WA 98225, USA; kenneth.rines@wwu.edu \\ Received 2014 August 13; accepted 2014 October 14; published 2014 December 5
}

\begin{abstract}
We use dense redshift surveys of nine galaxy clusters at $z \sim 0.2$ to compare the galaxy distribution in each system with the projected matter distribution from weak lensing. By combining 2087 new MMT/Hectospec redshifts and the data in the literature, we construct spectroscopic samples within the region of weak-lensing maps of high (70\%-89\%) and uniform completeness. With these dense redshift surveys, we construct galaxy number density maps using several galaxy subsamples. The shape of the main cluster concentration in the weak-lensing maps is similar to the global morphology of the number density maps based on cluster members alone, mainly dominated by red members. We cross-correlate the galaxy number density maps with the weak-lensing maps. The crosscorrelation signal when we include foreground and background galaxies at $0.5 z_{\mathrm{cl}}<z<2 z_{\mathrm{cl}}$ is $10 \%-23 \%$ larger than for cluster members alone at the cluster virial radius. The excess can be as high as $30 \%$ depending on the cluster. Cross-correlating the galaxy number density and weak-lensing maps suggests that superimposed structures close to the cluster in redshift space contribute more significantly to the excess cross-correlation signal than unrelated large-scale structure along the line of sight. Interestingly, the weak-lensing mass profiles are not well constrained for the clusters with the largest cross-correlation signal excesses ( $>20 \%$ for A383, A689, and A750). The fractional excess in the cross-correlation signal including foreground and background structures could be a useful proxy for assessing the reliability of weak-lensing cluster mass estimates.
\end{abstract}

Key words: cosmology: observations - dark matter - galaxies: clusters: individual (A267, A383, A611, A689, A697, A750, A963, RX J1720.1+2638, RX J2129.6+0005) - galaxies: kinematics and dynamics

Online-only material: color figures, machine-readable table

\section{INTRODUCTION}

Measurement of the mass distribution in galaxy clusters is an important test of structure formation models (Duffy et al. 2008; Prada et al. 2012; Rines et al. 2013). Among the many measurements of the mass distribution of clusters, only weak lensing (e.g., Hoekstra 2007; Okabe \& Umetsu 2008; Okabe et al. 2010; Umetsu et al. 2014) and the caustic method based on galaxy kinematics (Diaferio \& Geller 1997; Diaferio 1999; Serra et al. 2011) reliably measure the cluster mass distribution regardless of the cluster dynamical state. These measures can both extend into the infall region (Geller et al. 2013).

Weak lensing has grown into a powerful probe of the distribution of dark matter because it measures the total mass of a system directly regardless of the baryon content and/or dynamical state (Clowe et al. 2006; Huterer 2010; Shan et al. 2012). However, weak lensing includes the effect of structures projected along the line of sight (Hoekstra 2001); lensing provides a map of the total projected surface mass density. There are also several unresolved systematic errors in weak-lensing analysis, e.g., systematic uncertainties in the measurements of gravitational shear and in the photometric redshift estimation of the distribution of lensed sources (Huterer et al. 2013; Utsumi et al. 2014).

The signal in weak-lensing maps centered on a cluster is generally dominated by the cluster itself. However, there is an expected contribution to the signal from large-scale structures either associated or not associated with the cluster (Hoekstra
2001, 2003; Dodelson 2004). These structures are sometimes resolved in the weak-lensing maps and can often appear even within the virial radii of clusters. The lensing signal from these structures may introduce a bias and/or increase the uncertainty in a cluster mass estimate based on lensing (Hoekstra et al. 2011b; Becker \& Kravtsov 2011; Gruen et al. 2011; Bahé et al. 2012; Coe et al. 2012).

Galaxy redshift surveys provide a map of the threedimensional galaxy distribution. They can thus be used to resolve the structures along the line of sight that may contribute to the total projected mass (Hoekstra et al. 2011a; Geller et al. $2010,2013,2014 b$ ). Use of redshift surveys can also mitigate systematic errors resulting from the use of photometric redshifts in weak-lensing analysis (Coupon et al. 2013). A direct comparison of the structures identified in weak-lensing maps and in redshift surveys provides an important test of the issues limiting applications of weak lensing to measurement of cluster masses and mass profiles and to the identification of galaxy clusters (Geller et al. 2005, 2010; Kurtz et al. 2012; Utsumi et al. 2014; Starikova et al. 2014).

As an example of the impact of superimposed structure on a weak-lensing map, Geller et al. (2014a) used a deep, dense, nearly complete redshift survey of the strong-lensing cluster A383 to compare the galaxy distribution with the weaklensing results of Okabe et al. (2010). The weak-lensing map of A383 matches the galaxy number density map based on cluster members alone very well. However, a secondary peak in the weak-lensing map is not clearly visible in the galaxy number density map based on members (see their Figure 8). 
Table 1

List of Galaxy Clusters

\begin{tabular}{|c|c|c|c|c|c|c|c|c|c|}
\hline Name & $\begin{array}{l}\text { R.A.2000 } \\
\text { (deg) }\end{array}$ & $\begin{array}{l}\text { Decl.2000 } \\
\quad(\mathrm{deg})\end{array}$ & $z$ & $\begin{array}{l}\text { Source of } \\
\text { Redshifts }^{\mathrm{a}}\end{array}$ & $\begin{array}{c}\text { Number of } \\
z \text { Inside } \\
\text { Subaru FOV }\end{array}$ & $\begin{array}{c}\text { Completeness } \\
\text { Inside } \\
\text { Subaru FOV }\end{array}$ & $\begin{array}{l}\text { Subaru } \\
\text { FOV } \\
\text { (arcmin) }\end{array}$ & $\begin{array}{c}\text { Radius of } \\
\text { Entire Field } \\
\text { (arcmin) }\end{array}$ & $\begin{array}{c}\text { Number of } \\
\text { of } z \text { for } \\
\text { Entire Field }\end{array}$ \\
\hline A383 & 42.01417 & -3.52914 & 0.1887 & 1 & $411 / 153$ & $82 \%$ & $20^{\prime} \times 20^{\prime}$ & 51 & $2544 / 275$ \\
\hline A267 & 28.17485 & 1.00709 & 0.2291 & 2 & $419 / 154$ & $76 \%$ & $20^{\prime} \times 20^{\prime}$ & 33 & $1611 / 192$ \\
\hline A611 & 120.23675 & 36.05654 & 0.2880 & 3,4 & $335 / 129$ & $76 \%$ & $20^{\prime} \times 20^{\prime}$ & 37 & $1836 / 295$ \\
\hline A689 & 129.35600 & 14.98300 & 0.2789 & 2,5 & $333 / 119$ & $73 \%$ & $20^{\prime} \times 20^{\prime}$ & 33 & $1282 / 220$ \\
\hline A697 & 130.73982 & 36.36646 & 0.2812 & $2,5,6$ & $284 / 149$ & $89 \%$ & $16^{\prime} \times 16^{\prime}$ & 33 & $1152 / 269$ \\
\hline A750 & 137.24690 & 11.04440 & 0.1640 & 2,5 & $540 / 211$ & $73 \%$ & $24^{\prime} \times 24^{\prime}$ & 33 & $1344 / 305$ \\
\hline A963 & 154.26513 & 39.04705 & 0.2041 & $2,5,7$ & $318 / 161$ & $70 \%$ & $18^{\prime} \times 18^{\prime}$ & 33 & $1516 / 379$ \\
\hline RXJ1720.1+2638 & 260.04183 & 26.62557 & 0.1604 & 2,8 & $220 / 121$ & $89 \%$ & $14^{\prime} \times 14^{\prime}$ & 33 & $1511 / 349$ \\
\hline RX J2129.6+0005 & 322.41647 & 0.08921 & 0.2339 & 2,9 & $156 / 71$ & $71 \%$ & $12^{\prime} \times 12^{\prime}$ & 41 & $3522 / 249$ \\
\hline
\end{tabular}

Notes.

a (1) Geller et al. (2014a); (2) Rines et al. (2013); (3) K. J. Rines et al. 2014 (in preparation); (4) Lemze et al. (2013); (5) this study; (6) Girardi et al. (2006); (7) Jaffé et al. (2013); (8) Owers et al. (2011); (9) Drinkwater et al. (2010). We also add the redshifts from the SDSS DR10 and from NED.

b Number of redshifts/number of cluster members at $m_{\mathrm{r}, \text { Petro, } 0} \leqslant 20.5$.

${ }^{c}$ Number of redshifts/number of cluster members regardless of magnitude range.

A galaxy number density map that includes foreground and background galaxies around A383 produces a secondary peak consistent with the secondary weak-lensing peak. Thus, the secondary lensing peak apparently results from a superposition of foreground and background structures around A383. The secondary peak lies within the virial radius of A383 and the cluster mass profile can be affected by superimposed structures even within the virial radius. The pilot study of A383 demonstrates the importance of dense redshift surveys to understand the projected mass distribution revealed by weak lensing.

Here, we compare maps based on dense redshift surveys with weak-lensing maps for nine additional clusters. We compare the structures identified in the galaxy number density and weaklensing maps by cross-correlating the two. With this sample, we begin to quantify and elucidate the contribution to weak-lensing maps from structures superimposed along the line of sight.

Section 2 describes the cluster sample and the data including the weak-lensing maps and the deep redshift surveys. We measured 2087 new redshifts to obtain galaxy number density maps uniformly complete to $m_{r}=20.5$ for each cluster. We compare galaxy number density maps of galaxy clusters with the corresponding weak-lensing maps in Section 3. We discuss the results and conclude in Sections 4 and 5, respectively. Throughout, we adopt flat $\Lambda$ CDM cosmological parameters: $H_{0}=100 h \mathrm{~km} \mathrm{~s}^{-1} \mathrm{Mpc}^{-1}, \Omega_{\Lambda}=0.7$, and $\Omega_{m}=0.3$.

\section{DATA}

Among the 30 clusters at $z \sim 0.2$ with high-quality Subaru weak-lensing maps in Okabe et al. (2010), we select nine galaxy clusters with nearly complete redshift survey data. With the inclusion of redshifts measured in this study, all nine clusters have an overall spectroscopic completeness within the region of a weak-lensing map $>70 \%$ for $m_{\mathrm{r}, \text { Petro, } 0} \leqslant 20.5$.

Table 1 lists the nine clusters with the redshift source, the number of redshifts within the weak-lensing maps, the overall spectroscopic completeness within the weaklensing maps, the fields of view (FOVs) of the weaklensing maps, the size of the entire cluster field where we compile the redshift data, and the number of redshifts in the entire field. Here, we describe the data we use to compare the redshift surveys with the weak-lensing results.

\subsection{Weak-lensing Maps}

Okabe et al. (2010) use Subaru/Suprime-Cam images (Miyazaki et al. 2002) for $30 \mathrm{X}$-ray luminous galaxy clusters at $0.15 \leqslant z \leqslant 0.3$ to determine the mass distribution around clusters. Their paper includes a two-dimensional weak-lensing map of each cluster field. The map provides the normalized mass density field (i.e., the lensing convergence field, $\kappa$ ) relative to the $1 \sigma$ noise level expected from the intrinsic ellipticity noise. These maps are the basis for their derivation of projected mass distribution around each cluster.

Typical FOVs of the maps are $20^{\prime} \times 20^{\prime}$. They smooth the map with the Gaussian, typical FWHM of 1!2. The range of FWHM is $1.0-1.7$. We adopt their maps, listed in their Figures 16-45 (see their Appendix 3 for more details about the construction of the maps). Among the 30 clusters in their sample, we selected nine clusters where we measured new redshifts as necessary.

\subsection{The Cluster Redshift Surveys}

To compare the galaxy number density and weak-lensing map of a cluster, it is necessary to have a sufficiently dense, nearly complete redshift survey of the cluster (e.g., Geller et al. 2014a). Among the 30 clusters at $z \sim 0.2$ with Subaru weaklensing maps in Okabe et al. (2010), we first select five clusters with dense redshift data in the Hectospec Cluster Survey (HeCS; Rines et al. 2013). We supplement these data with redshifts from the literature (Girardi et al. 2006; Drinkwater et al. 2010; Owers et al. 2011; Lemze et al. 2013; Jaffé et al. 2013; Geller et al. 2014a), the Sloan Digital Sky Survey data release 10 (SDSS DR10; Ahn et al. 2014), and the NASA/IPAC Extragalactic Database (NED).

HeCS primarily observed galaxies close to the red sequence; because our goal here is to study all line-of-sight structure in the FOVs of the clusters, we require additional observations to obtain magnitude-limited redshift surveys. We made additional observations of four HeCS clusters (A689, A697, A750, and A963) in 2013 February and March with the 300 fiber Hectospec on the MMT $6.5 \mathrm{~m}$ telescope (Fabricant et al. 2005). The four clusters are within the footprint of the SDSS DR10. To obtain a high, uniform spectroscopic completeness at $m_{\mathrm{r}, \text { Petro, } 0} \leqslant$ 20.5 within the field of the weak-lensing map, we weighted the spectroscopic targets according to the galaxy apparent magnitude independent of color. 
Table 2

Redshifts in the Fields of A689, A697, A750, and A963

\begin{tabular}{|c|c|c|c|c|c|c|c|c|}
\hline Cluster & ID & $\begin{array}{l}\text { SDSS ObjID } \\
\text { (DR10) }\end{array}$ & $\begin{array}{l}\text { R.A.2000 } \\
\text { (deg) }\end{array}$ & $\begin{array}{l}\text { Decl.2000 } \\
\text { (deg) }\end{array}$ & $\begin{array}{c}m_{\mathrm{r}, \text { Petro, } 0} \\
(\mathrm{mag})\end{array}$ & $z$ & $\begin{array}{c}z \\
\text { Source }^{\mathrm{a}}\end{array}$ & Member $^{b}$ \\
\hline A689 & 1 & 1237667291574042910 & 128.795409 & 14.957459 & 18.939 & $0.00011 \pm 0.00003$ & 3 & 0 \\
\hline A689 & 2 & 1237667538535055415 & 128.801168 & 15.053992 & 20.484 & $0.52506 \pm 0.00018$ & 3 & 0 \\
\hline A689 & 3 & 1237667538535055402 & 128.802527 & 15.004511 & 17.237 & $0.00009 \pm 0.00001$ & 3 & 0 \\
\hline A689 & 4 & 1237667538535055664 & 128.808520 & 15.046711 & 17.286 & $0.13889 \pm 0.00003$ & 3 & 0 \\
\hline A689 & 5 & 1237667291574042897 & 128.812113 & 14.875166 & 17.978 & $0.00021 \pm 0.00001$ & 3 & 0 \\
\hline A689 & 6 & 1237667538535055646 & 128.816686 & 15.004262 & 19.290 & $-0.00004 \pm 0.00005$ & 3 & 0 \\
\hline A689 & 7 & 1237667291574042924 & 128.825021 & 14.912634 & 17.442 & $0.15485 \pm 0.00003$ & 3 & 0 \\
\hline A689 & 8 & 1237667538535055723 & 128.827412 & 15.141970 & 17.646 & $0.15470 \pm 0.00004$ & 3 & 0 \\
\hline A689 & 9 & 1237667538535055734 & 128.830726 & 15.171179 & 17.600 & $0.16997 \pm 0.00003$ & 3 & 0 \\
\hline A689 & 10 & 1237667291574042958 & 128.839568 & 14.954218 & 17.674 & $0.00081 \pm 0.00001$ & 3 & 0 \\
\hline
\end{tabular}

Notes.

a (1) This study; (2) Rines et al. (2013); (3) SDSS DR10; (4) Girardi et al. (2006); (5) Jaffé et al. (2013); (6) NED.

b (0) Cluster non-members; (1) Cluster members.

(This table is available in its entirety in a machine-readable form in the online journal. A portion is shown here for guidance regarding its form and content.)

We used the 270 line $\mathrm{mm}^{-1}$ grating of Hectospec that provides a dispersion of $1.2 \AA$ pixel $^{-1}$ and a resolution of $\sim 6 \AA$. We used $3 \times 20$ minute exposures for each field, and obtained spectra covering the wavelength range 3650-9150 A. During the pipeline processing, spectral fits are assigned a quality flag of "Q" for high-quality redshifts, "?" for marginal cases, and "X" for poor fits. We use only the spectra with reliable redshift measurements (i.e., "Q"). We set up two or three different Hectospec fields for each cluster and obtained 470-610 reliable redshifts per cluster.

Table 2 lists the galaxy redshift data in the fields of the clusters. We list 5294 galaxies with measured redshifts, including 2087 new Hectospec redshifts for four clusters (A689, A697, A750, and A963). The table contains the cluster name, identification, SDSS DR10 ObjID, the right ascension (R.A.), declination (decl.), $r$-band Petrosian magnitude with Galactic extinction correction (from the SDSS DR10), the redshift $(z)$ and its error, the redshift source, and the cluster membership flag.

Figure 1 shows Galactic extinction corrected (denoted by the subscript, "0") $(g-r)_{\text {model }, 0}-m_{\mathrm{r}, \text { Petro, } 0}$ color-magnitude diagram for each cluster. Black dots and green squares are extended sources without and with measured redshifts, respectively. Red circles and blue crosses are red and blue cluster member galaxies. Obviously, most bright galaxies have measured redshifts. The plot includes all the sources in the entire cluster field (see Table 1 for the field size), including the galaxies outside the weak-lensing maps, thus there are some bright galaxies without measured redshifts. We explain the details of the spectroscopic completeness in the region covered by each weak-lensing map in the next section.

To determine the membership of galaxies in each cluster, we use the caustic technique (Diaferio \& Geller 1997; Diaferio 1999; Serra et al. 2011), originally devised to determine the mass profiles of galaxy clusters. The technique produces a useful tool for determining cluster membership. Analysis of galaxy clusters in a cosmological $N$-body simulation indicates that the caustic technique identifies true cluster members with $95 \%$ completeness within $3 r_{200}$. The contamination of interlopers in the member galaxy catalog is only $2 \%-8 \%$ at $1-3 r_{200}$ (Serra \& Diaferio 2013).

The caustic technique first uses the redshifts and the position on the sky of the galaxies to determine a hierarchical center of the cluster based on a binary tree analysis. We then plot the rest-frame clustercentric velocities of galaxies as a function of projected clustercentric radius centered on the hierarchical center. Figure 2 shows this phase-space diagram for each cluster; the expected trumpet-shaped pattern is obvious (Kaiser 1987; Regos \& Geller 1989). The caustics (solid lines) generally agree with the lines based on a visual impression. They are cleanly defined, especially at small radii. We use all the galaxies with measured redshifts regardless of their magnitudes for determining the membership but restrict our analysis to the galaxies at $m_{\mathrm{r}, \text { Petro, } 0} \leqslant 20.5$ within the weak-lensing maps for comparison between the galaxy number density and weaklensing maps.

To segregate the red and blue cluster populations, we define a red sequence from a linear fit to the bright, red member galaxies in Figure 1 (e.g., $m_{\mathrm{r} \text {,Petro, } 0}<19.5$ and $1<(g-r)_{\text {model }, 0}<2$ for A383). The solid line in each panel shows the red sequence for each cluster. The typical rms scatter $(\sigma)$ around the red sequence is $\sim 0.1 \mathrm{mag}$. A line $3 \sigma$ blueward of the red sequence separates the red and blue members.

When we construct galaxy number density maps in Section 3.1, we also use SDSS photometric redshifts for galaxies without spectroscopic redshifts (Csabai et al. 2003). The rms uncertainties of photometric redshifts for the galaxies at $m_{r}<18$ and $m_{r}<21$ are $\sim 0.035$ and $\sim 0.103$, respectively (Csabai et al. 2003). The redshift range necessary for constructing galaxy number density maps to be compared with weaklensing maps is broad enough not to be significantly affected by these uncertainties.

As a prototypical example, we compare spectroscopic and photometric redshifts for galaxies in the field of A383 in the left panel of Figure 3. As expected, the photometric redshifts roughly agree with the spectroscopic redshifts with a large scatter. The top right panel shows $r$-band Petrosian magnitudes of galaxies with spectroscopic (red filled circles) and photometric (open circles) redshifts as a function of redshift. The bottom right panel shows the redshift histogram for each galaxy sample (red hatched and open histograms for spectroscopic and photometric redshifts, respectively). The foreground and background structures at $z \sim 0.14$ and $z \sim 0.3$, respectively $\left(z_{\mathrm{A} 383}=0.1887\right)$, are apparent in the histogram. As expected, the photometric redshifts contribute little; they are important mainly for faint galaxies. The comparison of spectroscopic and 


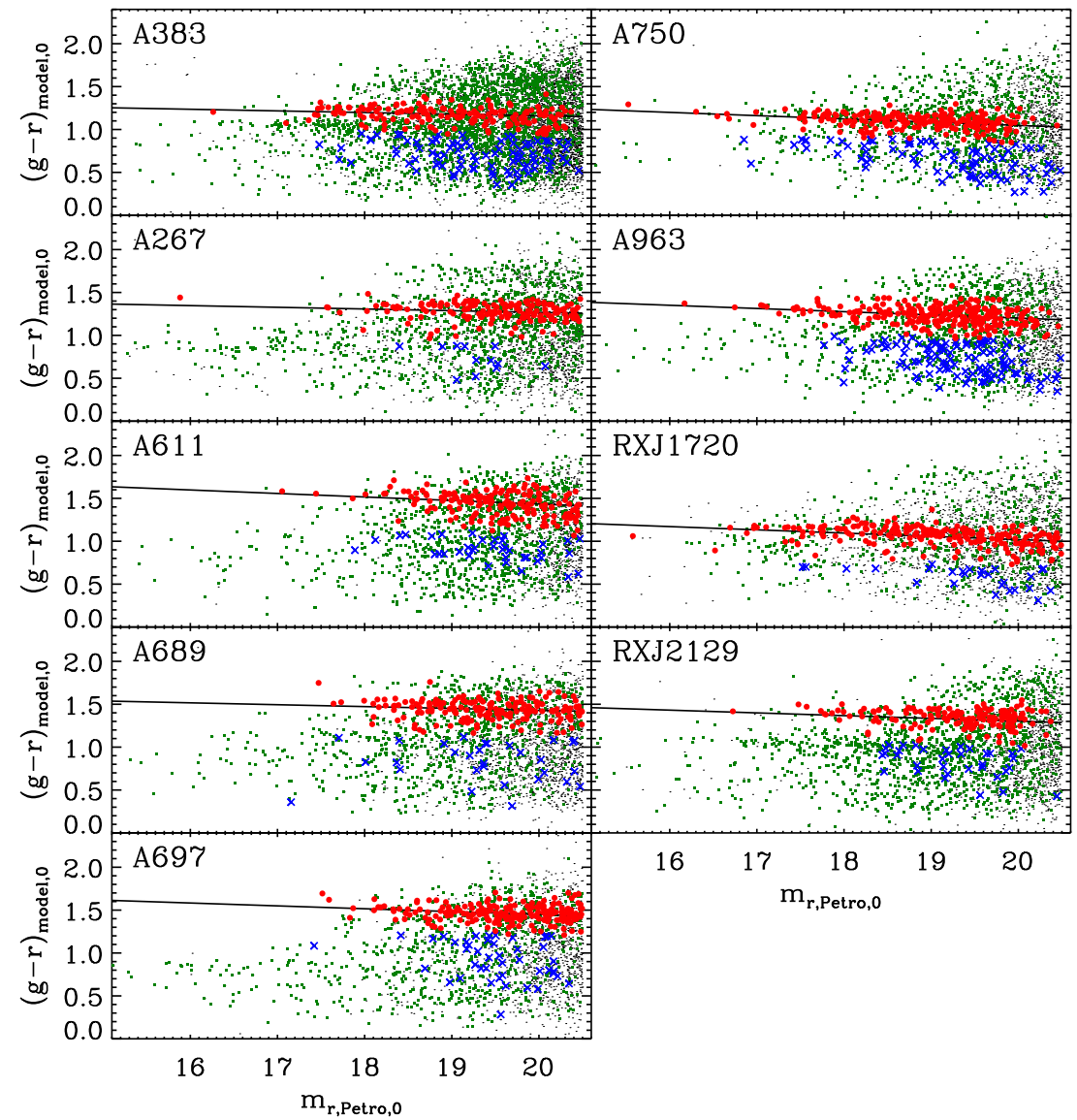

Figure 1. Color-magnitude diagram for galaxies in the fields of nine galaxy clusters. Black dots and green squares are galaxies without and with spectroscopic redshifts, respectively. Red circles and blue crosses are red and blue member galaxies with spectroscopic redshifts, respectively. The solid line is the best fit of the red sequence in each cluster.

(A color version of this figure is available in the online journal.)

photometric redshifts for the other eight clusters is similar to A383; thus, we do not display them.

\section{RESULTS}

Here, we construct galaxy number density maps using several galaxy subsamples in Section 3.1 and cross-correlate them with the weak-lensing maps in Section 3.2. We first test whether red cluster members can reproduce the main weak-lensing peak (see, e.g., Zitrin et al. 2010), and then add other populations successively (i.e., foreground/background galaxies along the line of sight) to gauge their impact on the weak-lensing maps.

\subsection{Galaxy Number Density Maps}

To construct galaxy number density maps based on a spectroscopic sample of galaxies, it is important to understand any bias introduced by the spectroscopic observations, especially spectroscopic incompleteness. The pixel size and smoothing scale we need are set by the weak-lensing maps of Okabe et al. (2010), e.g., $201 \times 201$ pixels for the $20^{\prime} \times 20^{\prime}$ weak-lensing map and Gaussian smoothing scale of FWHM $=1$ '.2 for A383.

\subsubsection{Spectroscopic Completeness}

Panel (a) of Figure 4 shows a two-dimensional map of the spectroscopic completeness for $m_{\mathrm{r}, \text { Petro, } 0} \leqslant 20.5$ as a function of R.A. and decl., matched to the FOV of the weak-lensing map of A383. The two-dimensional completeness map is in
$9 \times 9$ pixels for the $20^{\prime} \times 20^{\prime}$ weak-lensing map. Panels (b) and (c) show the integrated completeness as a function of R.A. and decl., respectively. The overall completeness in this field is $\sim 81 \%$. Although there are three pixels with a completeness $<50 \%$, the completeness changes little with R.A. and decl. Panel (d) shows the integrated completeness as a function of $r$-band magnitude; it drops only at $m_{\mathrm{r}, \text { Petro, } 0}>20$.

We also show the spatial distribution of galaxies superimposed on the A383 weak-lensing map of Okabe et al. (2010) in panel (e). Many member galaxies of A383 (open circles) are distributed around the peaks of the weak-lensing map, but some foreground and background galaxies (squares) are located around the peaks. The plots for other clusters are in the Appendix.

\subsubsection{Construction of Galaxy Number Density Maps: Revisiting A383}

To study the relative contribution of cluster and foreground/ background structure to the weak-lensing maps, we construct an extensive set of galaxy number density maps based on several galaxy subsamples. We smooth the contours with the same Gaussian FWHM as for the weak-lensing map of Okabe et al. (2010), e.g., FWHM = 1'.2 for A383. We also use the same pixel size as for the weak-lensing map (e.g., $201 \times 201$ pixels for $20^{\prime} \times 20^{\prime} \mathrm{FOV}$ of A383). Figure 5 shows these number density maps (blue contours) superimposed on the weak-lensing map (gray contours). The top panels are based on cluster member galaxies alone (all, red, and blue members from left to right). 


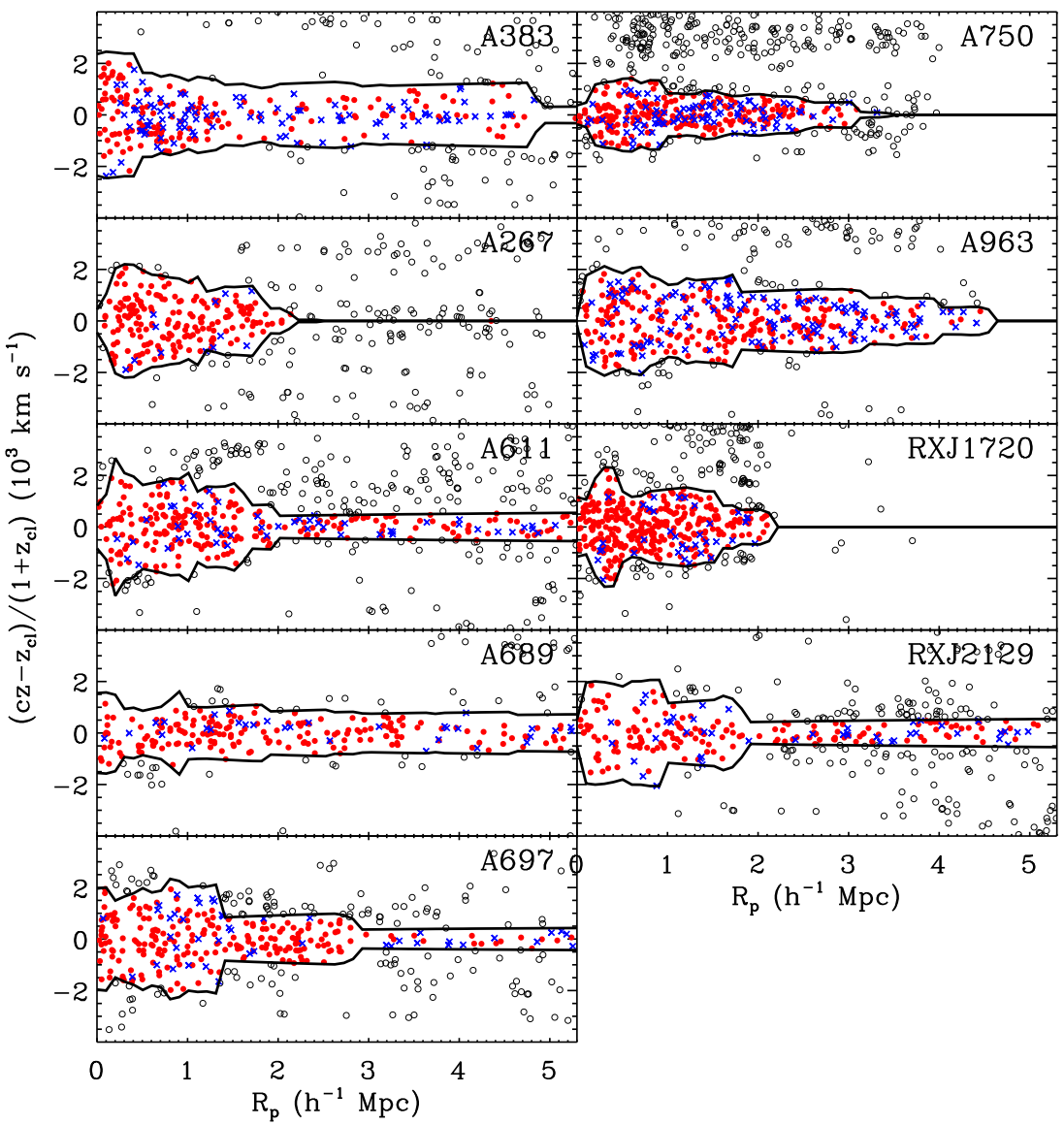

Figure 2. Redshift (rest-frame clustercentric velocity) diagram for the nine clusters in this study. Red circles and blue crosses are red and blue member galaxies, respectively. Open circles are non-member galaxies. Black lines are the caustics that distinguish member and non-member galaxies. (A color version of this figure is available in the online journal.)
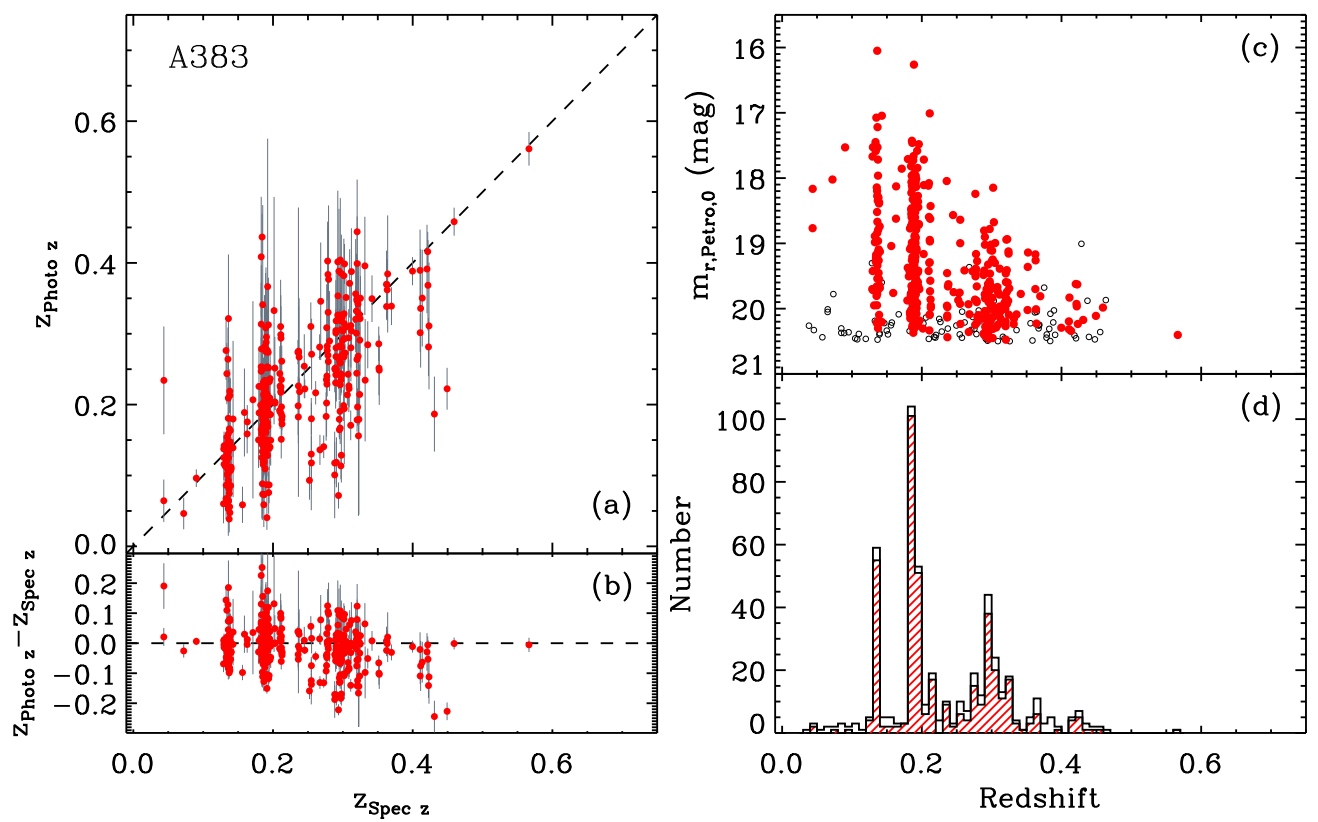

Figure 3. (a)-(b) Comparison between photometric redshifts (Csabai et al. 2003) and spectroscopic redshifts for the galaxies with $m_{\mathrm{r}, \text { Petro, } 0}<20.5$ inside the field of the weak-lensing map (see Figure 4). (c) $r$-band Petrosian magnitudes of galaxies with spectroscopic (red filled circles) and photometric (open circles) redshifts as a function of redshift. (d) Redshift distribution of galaxies with spectroscopic (red hatched histogram) and photometric (open histogram) redshifts. (A color version of this figure is available in the online journal.) 

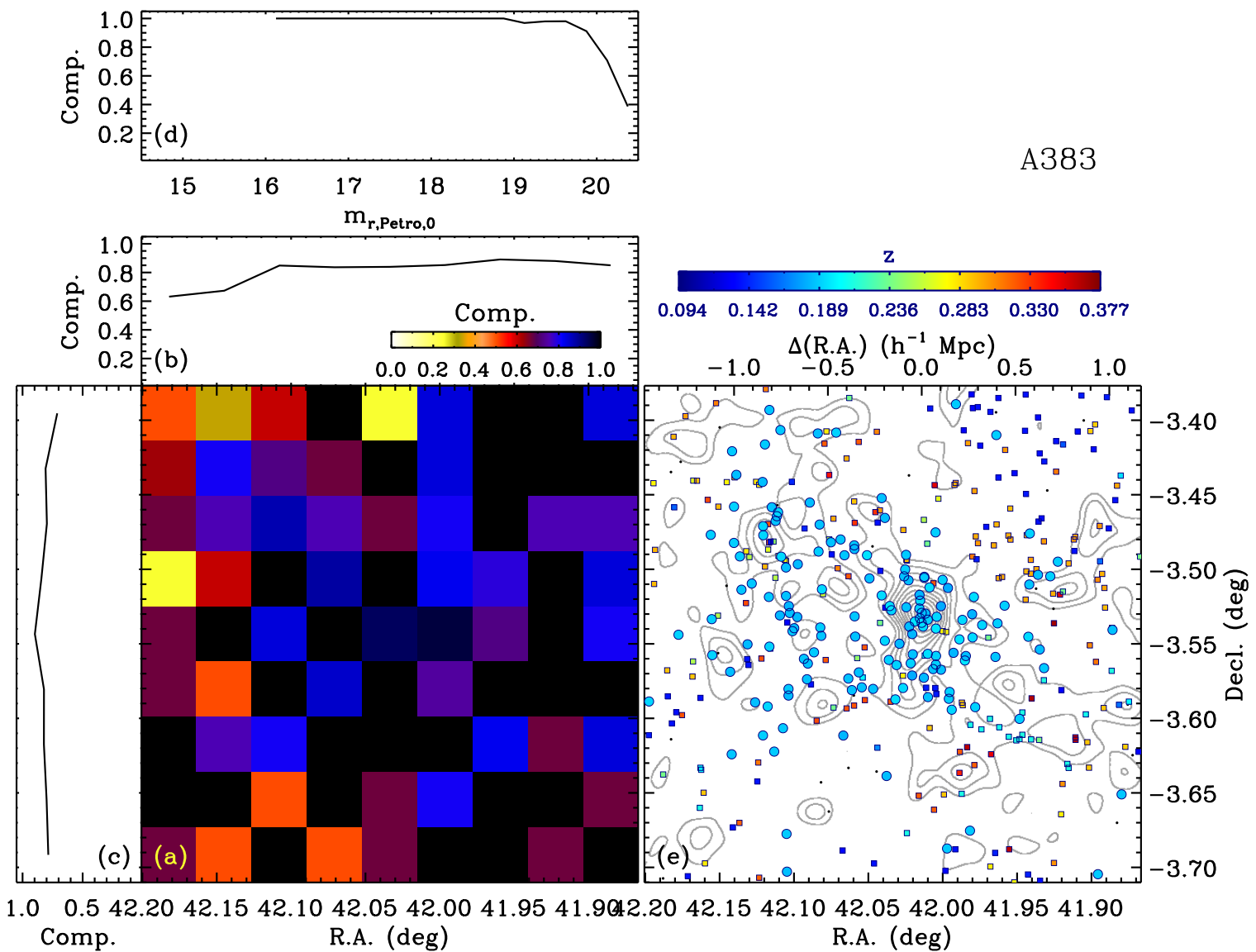

Figure 4. (a) Two-dimensional spectroscopic completeness in the field of A383 as a function of right ascension and of declination. Integrated spectroscopic completeness as a function of (b) right ascension, (c) declination, and (d) $r$-band magnitude. (e) Spatial distribution of galaxies at $m_{\mathrm{r}, \text { Petro, } 0} \leqslant 20.5$ (open circles: members, squares: non-members inside the color bar redshift window, dots: non-members outside the color bar redshift window). Gray contours are the weak-lensing map of Okabe et al. (2010).

(A color version of this figure is available in the online journal.)

We separate red and blue galaxies based on their positions in the $(g-r)_{\text {model }, 0}-m_{\mathrm{r}, \text { Petro, } 0}$ color-magnitude diagram (see Section 2.2).

As in Geller et al. (2014a), the global morphology of the spatial distribution of cluster members alone (red and red plus blue populations) is similar to the shape of the main concentration in the weak-lensing map; both show a north-south elongation (see panel (a)). The density map based on red members (panel (b)) is also similar to the cluster shape in the weak-lensing map. Interestingly, blue members alone have little correspondence with the weak-lensing map (panel (c)). This comparison supports the idea that the red population provides a reasonable tracer of the mass distribution of a galaxy cluster provided that the selection is dense and broad enough around the red sequence (Rines et al. 2013; Geller et al. 2014a). The comparison also suggests that the assumption that red cluster galaxies trace the dark matter distribution is a very reasonable approach for strong-lensing models (Broadhurst et al. 2005; Zitrin et al. 2009; Medezinski et al. 2010).

The middle panels show contours for galaxies in the redshift range $0.5 z_{\mathrm{cl}} \leqslant z \leqslant 2 z_{\mathrm{cl}}$ (i.e., $0.094 \leqslant z \leqslant 0.377$ for A383) where the contribution to the lensing signal may be significant (Hu 1999). The number density map in panel (d) is based on galaxies with measured redshifts. To account for galaxies without measured redshifts, we use two methods to construct the maps; we correct statistically for spectroscopic incompleteness, and we use photometric redshifts. To correct for spectroscopic incompleteness, we compute the spectroscopic completeness for each object with a measured redshift in a three-dimensional parameter space: $r$-band magnitude, R.A., and decl. The left panels in Figure 4 show the spectroscopic completeness in this parameter space. We thus weight each galaxy by the inverse of the spectroscopic completeness to derive the galaxy number density map and show it in panel (e). We also use SDSS photometric redshifts for the galaxies without spectroscopic redshifts (see Section 2.2 for details). The corresponding number density map is given in panel (f).

In the bottom panels, we use the same galaxy samples as in the middle panels, but, additionally, we weight each galaxy with the stellar mass. We compute stellar masses using the SDSS fiveband photometric data with the Le Phare ${ }^{6}$ code (Arnouts et al. 1999; Ilbert et al. 2006). Details of the stellar mass estimates are in Zahid et al. (2014). Three panels show the maps based on galaxy samples of observed galaxies (panel $(\mathrm{g})$ ), weighted by spectroscopic completeness (panel (h)), and supplemented by photometric redshifts (panel (1)).

It is interesting that the global morphology of the number density maps in panels (d)-(1) is similar despite the different correction methods. No matter how we weight the data, the correspondence between the galaxy number density and weaklensing maps is more remarkable when we include foreground and background galaxies in the galaxy number density maps

\footnotetext{
$\overline{6}$ http://www.cfht.hawaii.edu/ arnouts/lephare.html
} 
(a) All members $(0.179<\mathrm{z}<0.197)$

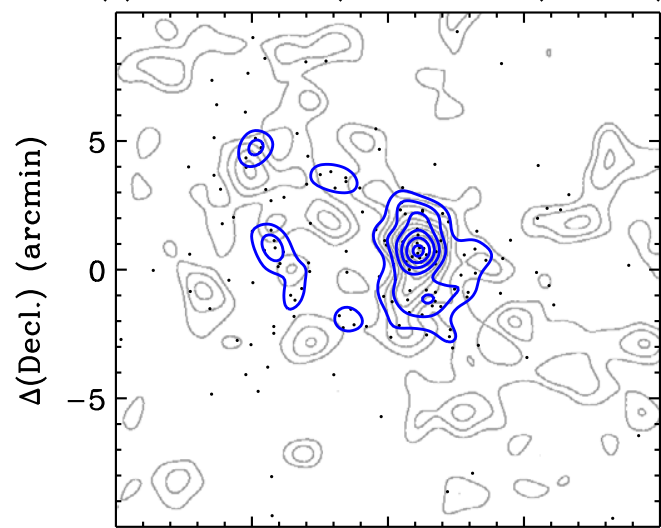

(d) Gals @ $0.094<z<0.377$

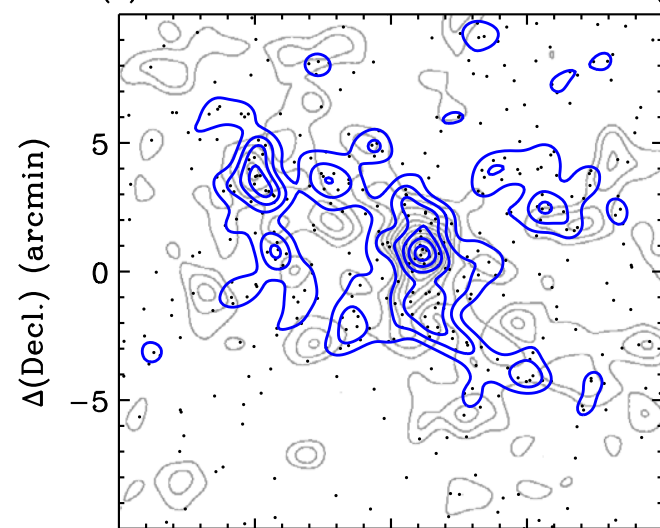

(b) Red members $(0.179<\mathrm{z}<0.197)$

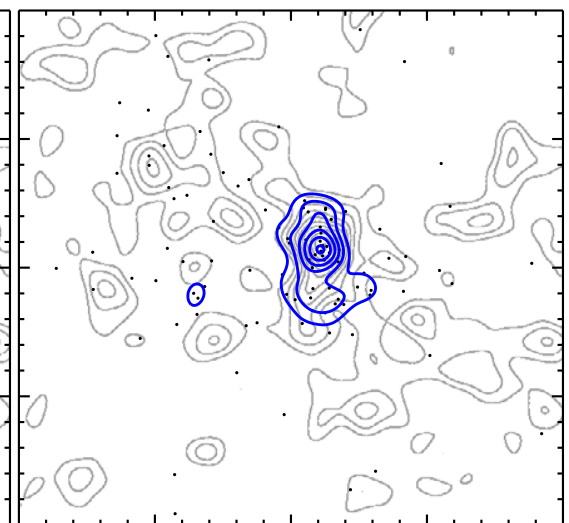

(e) Gals @ $0.094<z<0.377$, specW

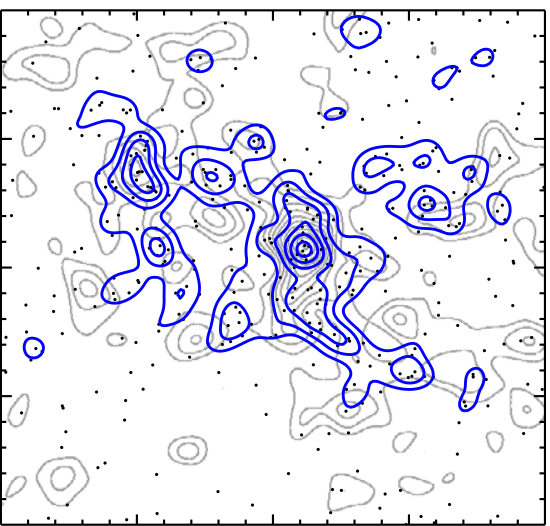

(f) Gals @ 0.094<z<0.377, photz

c) Blue members $(0.179<\mathrm{z}<0.197)$
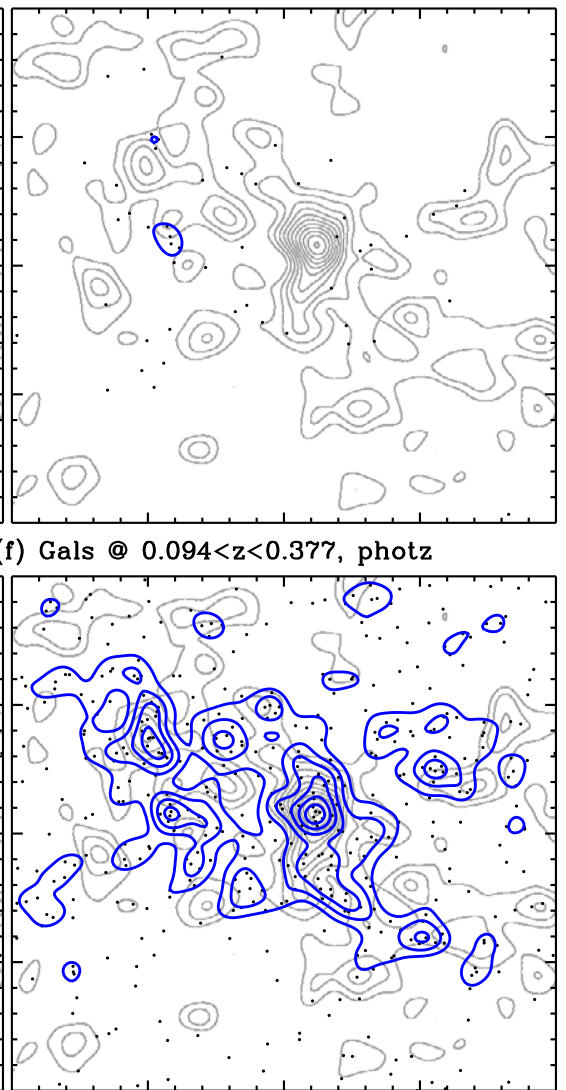

(g) Gals @ $0.094<z<0.377$, M.W

(h) Gals @ 0.094<z<0.377, specW \& M.W (i) Gals @ $0.094<z<0.377$, photz \& M.W
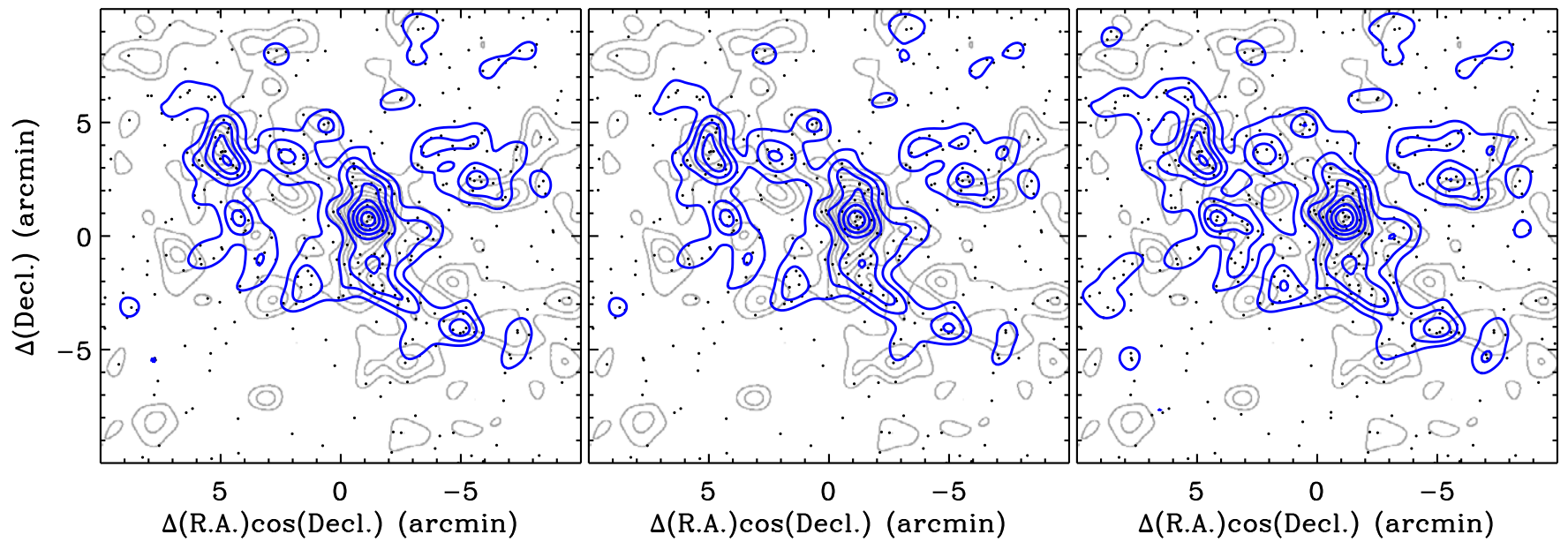

Figure 5. A383 galaxy number density maps for several subsamples at $m_{\mathrm{r}, \text { Petro, } 0}<20.5$. The lowest cluster surface number density contour is 1.61 galaxies arcmin ${ }^{-2}$, and the contours increase in steps of 0.85 galaxy arcmin ${ }^{-2}$. We smooth the contours with the Gaussian of FWHM $=1.2$, the same smoothing scale as for the weak-lensing maps of Okabe et al. (2010). The top row is for (a) all cluster members, (b) red cluster members, and (c) blue cluster members. The middle row is for the galaxies in the redshift range $0.5 z_{\mathrm{cl}} \leqslant z \leqslant 2 z_{\mathrm{cl}}$ (i.e., $0.094<z<0.377$ for A383): (d) without any weights, (e) weighted by spectroscopic completeness, and (f) complemented by galaxies with photometric redshifts. The bottom row is for the same sample as for in the middle row, but weighted by stellar masses of galaxies as well: $(\mathrm{g})$ without any weights, (h) weighted by spectroscopic completeness, and (1) complemented by galaxies with photometric redshifts.

(A color version of this figure is available in the online journal.)

(panels (d)-(1)), underscoring the contribution of large-scale structure along the line of sight to the weak-lensing signal.

\subsection{Cross-correlation between Galaxy Number Density and Weak-lensing Maps}

Here, we cross-correlate the weak-lensing and galaxy number density maps for several of the galaxy subsamples. We use the normalized cross-correlation (NCC), widely used in image processing (Gonzalez \& Woods 2002). It is defined by

$$
\operatorname{NCC}(x, y)=\frac{\sum_{i, j} I_{1}(i, j) I_{2}(i+x, j+y)}{\sqrt{\sum_{i, j} I_{1}^{2}(i, j)} \sqrt{\sum_{i, j} I_{2}^{2}(i+x, j+y)}},
$$

where $I_{1}(i, j)$ and $I_{2}(i, j)$ are pixel values of the galaxy number density and weak-lensing maps, respectively. 

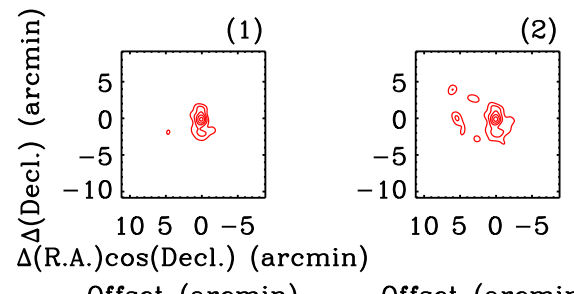

(2)
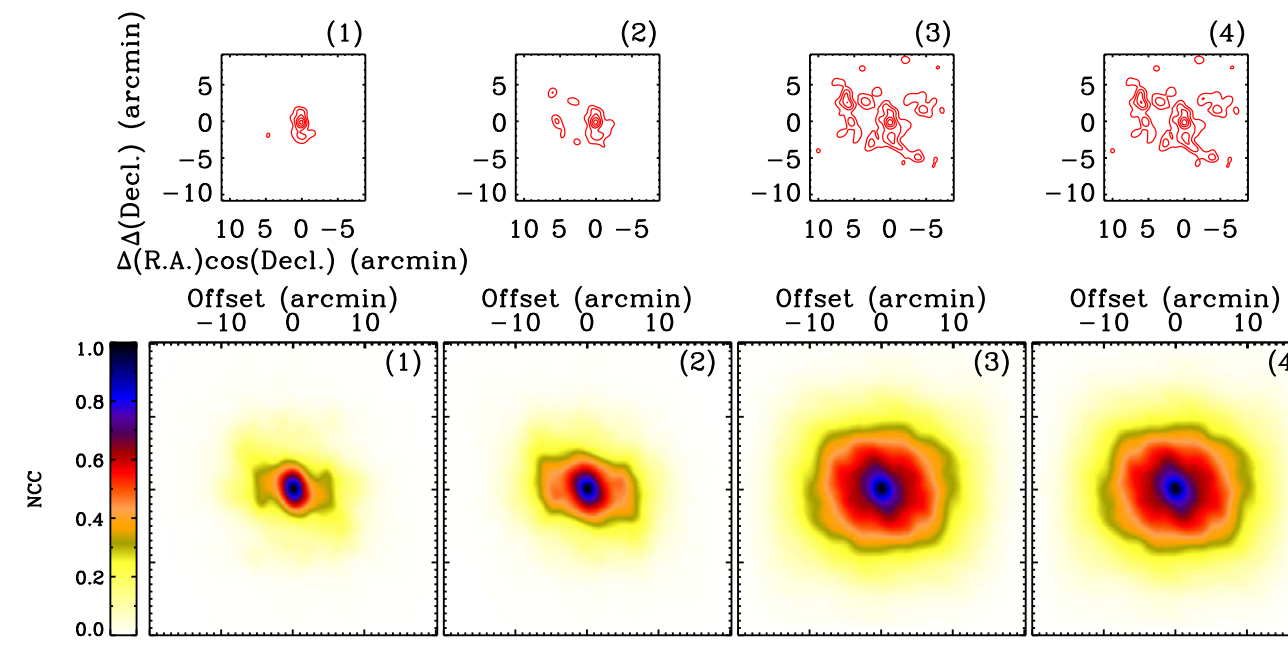

(2)

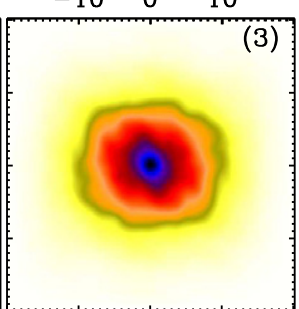

3) $]$
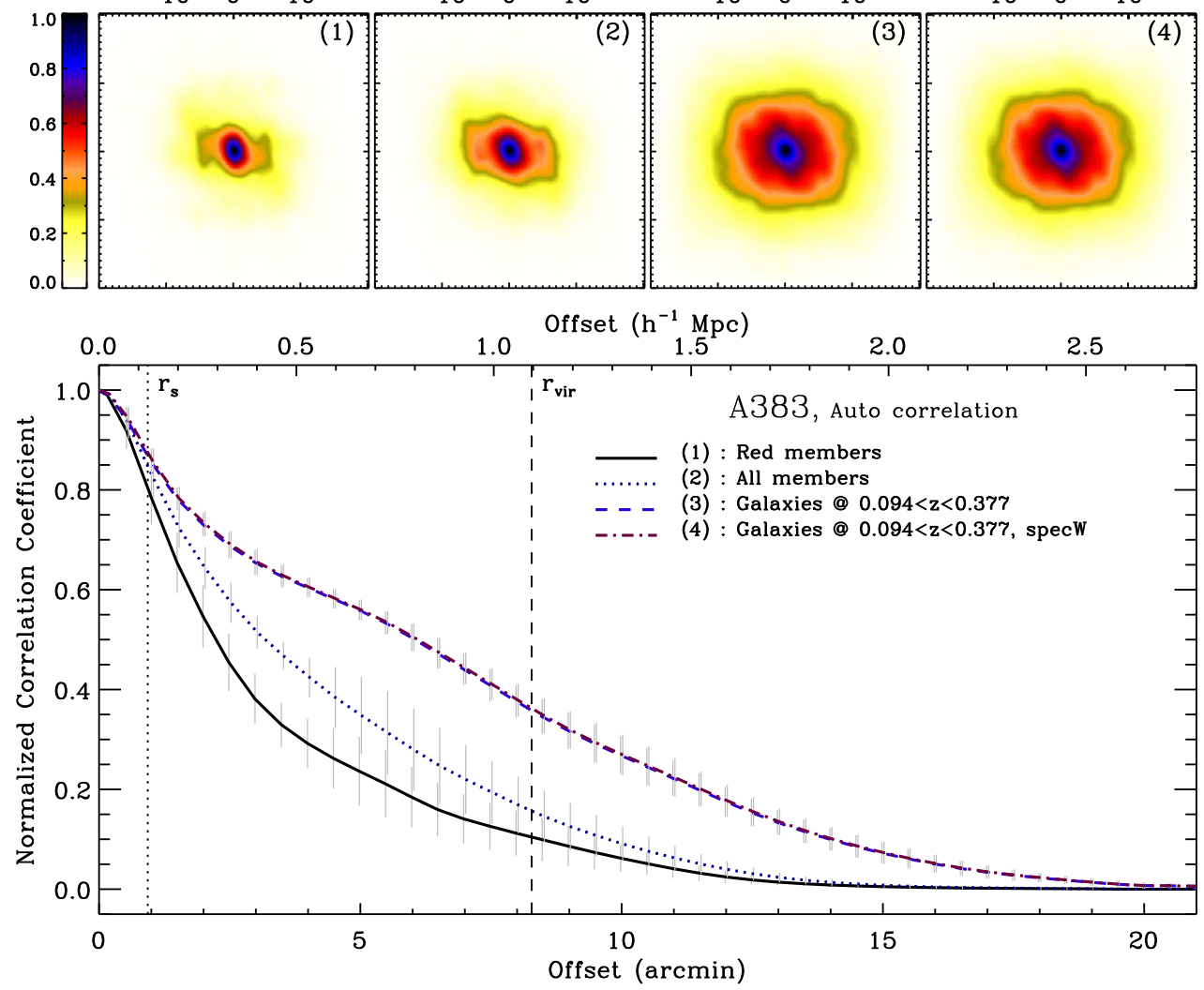

Figure 6. Normalized autocorrelation of A383 galaxy number density maps. Top: red contours indicate galaxy number density maps for four subsamples. Middle: two-dimensional normalized autocorrelation maps. Bottom: azimuthally averaged correlation signal as a function of offset. Vertical dotted and dashed lines indicate $r_{s}$ and $r_{\text {vir }}$ from Okabe et al. (2010), respectively.

(A color version of this figure is available in the online journal.)

\subsection{1. $A 383$}

To test our code and to understand what we can learn from the cross-correlation, we first calculate the autocorrelation for a galaxy number density map for a simulated cluster. We construct a mock galaxy catalog with 1000 galaxies in a cluster following the Navarro-Frenk-White profile (NFW; Navarro et al. 1997). We use the NFW profile of A383 with $r_{200}=1.184\left(h^{-1} \mathrm{Mpc}\right)$ and $c_{200}=6.51$ derived in Newman et al. (2013), and we show the results in the Appendix. As expected, the normalized autocorrelation signal is equal to unity at zero offset, and the correlation signal decreases with offset. It converges to zero at large radius (i.e., $r>r_{200}$ ); in other words, there is intrinsically no autocorrelation signal at this radius and/or, the autocorrelation signal becomes small because the radius reaches the edge of the maps.

We also calculate the autocorrelation for galaxy number density maps representing several galaxy subsamples of A383 and show the results in Figure 6. The top panels show the galaxy number density maps (red contours) for four subsamples: (1) red members, (2) all members, (3) galaxies at $0.5 z_{\mathrm{cl}} \leqslant z \leqslant 2 z_{\mathrm{cl}}$, and (4) galaxies at $0.5 z_{\mathrm{cl}} \leqslant z \leqslant 2 z_{\mathrm{cl}}$ weighted by their spectroscopic completeness (see Figure 4). The middle panels show the two-dimensional autocorrelation map for each case. The bottom panel shows the azimuthally averaged autocorrelation signal derived from each of the middle panels. The gray error bar indicates the dispersion in the two-dimensional correlation signal at each offset. As expected, the normalized correlation signal is unity at zero offset. The autocorrelation signal converges to zero at $r>r_{\text {vir }}$ for cases based on members alone (solid and dotted lines). However, the signal for cases including the galaxies at $0.5 z_{\mathrm{cl}} \leqslant z \leqslant 2 z_{\mathrm{cl}}$ (dashed and dot-dashed lines) is not negligible, even at $r \sim r_{\text {vir }}$, and decreases slowly; this effect results mainly from foreground and background galaxies along the line of sight that contribute to the autocorrelation signal.

Figure 7 shows the results of cross-correlating the galaxy number density maps and weak-lensing map. As in Figure 6, the top panels show the galaxy number density maps (red contours) for four galaxy subsamples superimposed on the weak-lensing maps (gray contours): (1) red members, (2) all members, (3) galaxies at $0.5 z_{\mathrm{cl}} \leqslant z \leqslant 2 z_{\mathrm{cl}}$, and (4) galaxies at $0.5 z_{\mathrm{cl}} \leqslant z \leqslant 2 z_{\mathrm{cl}}$ weighted by their spectroscopic completeness 


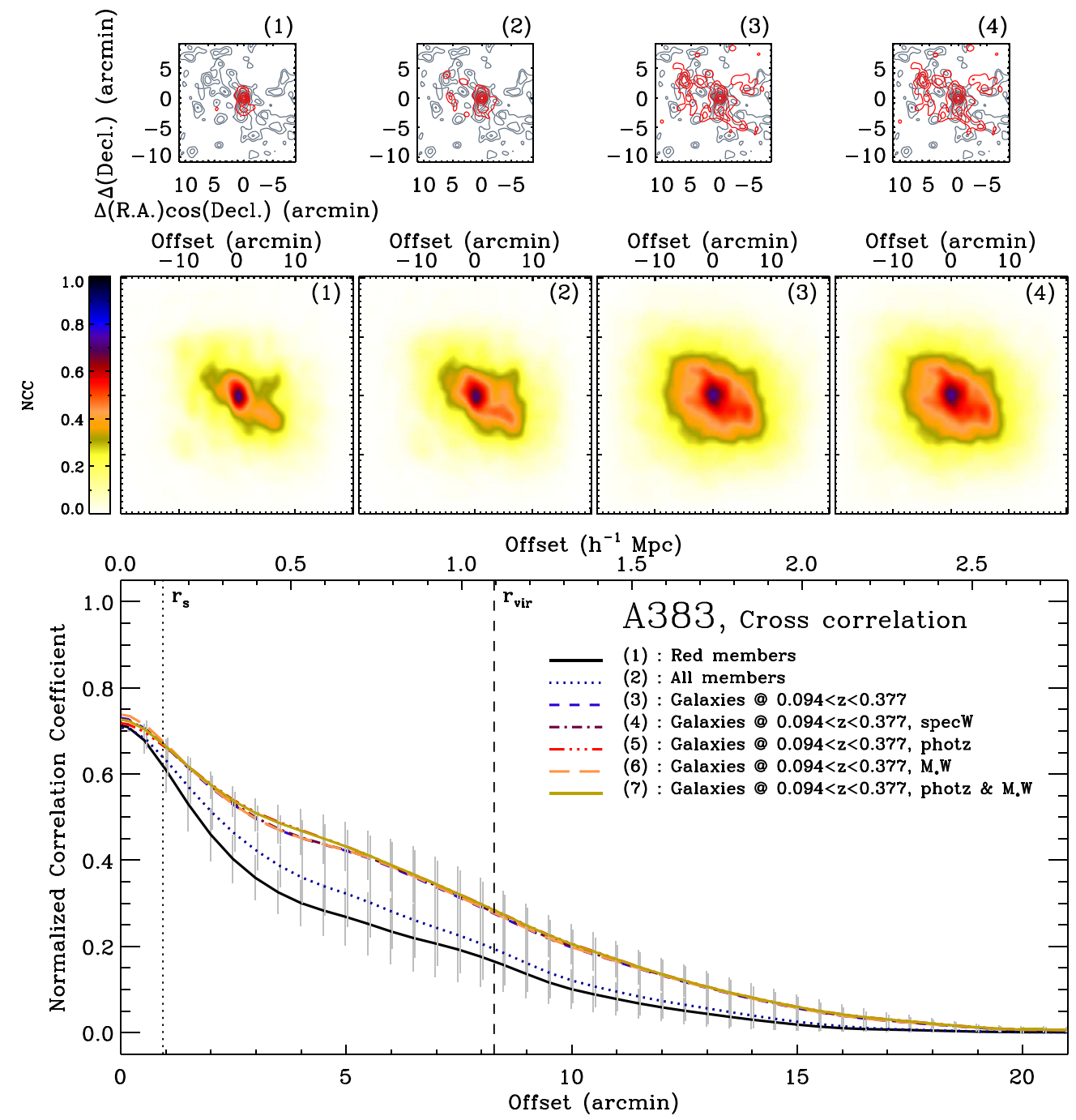

Figure 7. Normalized cross-correlation of A383 galaxy number density maps. Top: gray and red contours indicate weak-lensing and galaxy number density maps, respectively. We use seven galaxy subsamples listed in the bottom panel but show only four examples. Middle: two-dimensional normalized cross-correlation maps. Bottom: azimuthally averaged correlation signal as a function of offset. Vertical dotted and dashed lines indicate $r_{s}$ and $r_{\mathrm{vir}}$ from Okabe et al. (2010), respectively.

(A color version of this figure is available in the online journal.)

(see Figure 4). The middle panels show the two-dimensional cross-correlation map for each case. We use seven galaxy subsamples in the cross-correlation but show only four cases in the top and middle panels for ease of view. The bottom panel shows the azimuthally averaged cross-correlation signal for the seven galaxy subsamples.

Case (1) based on red member galaxies gives, not surprisingly, the narrowest correlation peak. The signal converges to zero at $r>r_{\text {vir }}$ for the cases based on member galaxies alone (solid and dotted lines). The cases including galaxies at $0.5 z_{\mathrm{cl}} \leqslant z \leqslant 2 z_{\mathrm{cl}}$ (i.e., cases 3-7) are similar to one another and show a broader distribution. The cross-correlation signal is always larger than for the cases based on member galaxies alone, indicating the non-negligible contribution of foreground and background galaxies to the weak-lensing map.

\subsubsection{Comments on Individual Clusters}

Here, we discuss the cross-correlation results for the other eight clusters. The plots for the eight clusters are given in Figures 8-11 (see Figure 7 for A383).
A267. The peak of the galaxy number density map based on members alone (top left panels) seems to be offset by $\sim 2^{\prime}$ from the central peak of the lensing map, but the two peaks coincide when we include foreground and background galaxies in the number density map. The number density maps based on members alone show an overdensity only in the central region. When we include foreground and background galaxies in the map, several weak-lensing peaks other than the cluster appear in the number density map. Thus, the normalized cross-correlation signal including foreground and background galaxies exceeds those based on cluster members alone at zero offset. This excess can indicate a non-negligible contribution of foreground and background galaxies to the weak-lensing signal, but Okabe et al. (2010) found nothing unusual in deriving the weak-lensing mass profile for this cluster.

A611. This cluster is the most distant cluster $(z=0.288)$ in the sample. The central region of the weak-lensing map shows a north/east-south/west elongation. The number density contours based on member galaxies alone weakly follow this elongation. However, the elongated contours are more similar to the weak-lensing contours when we include foreground and background galaxies. In other words, foreground and 


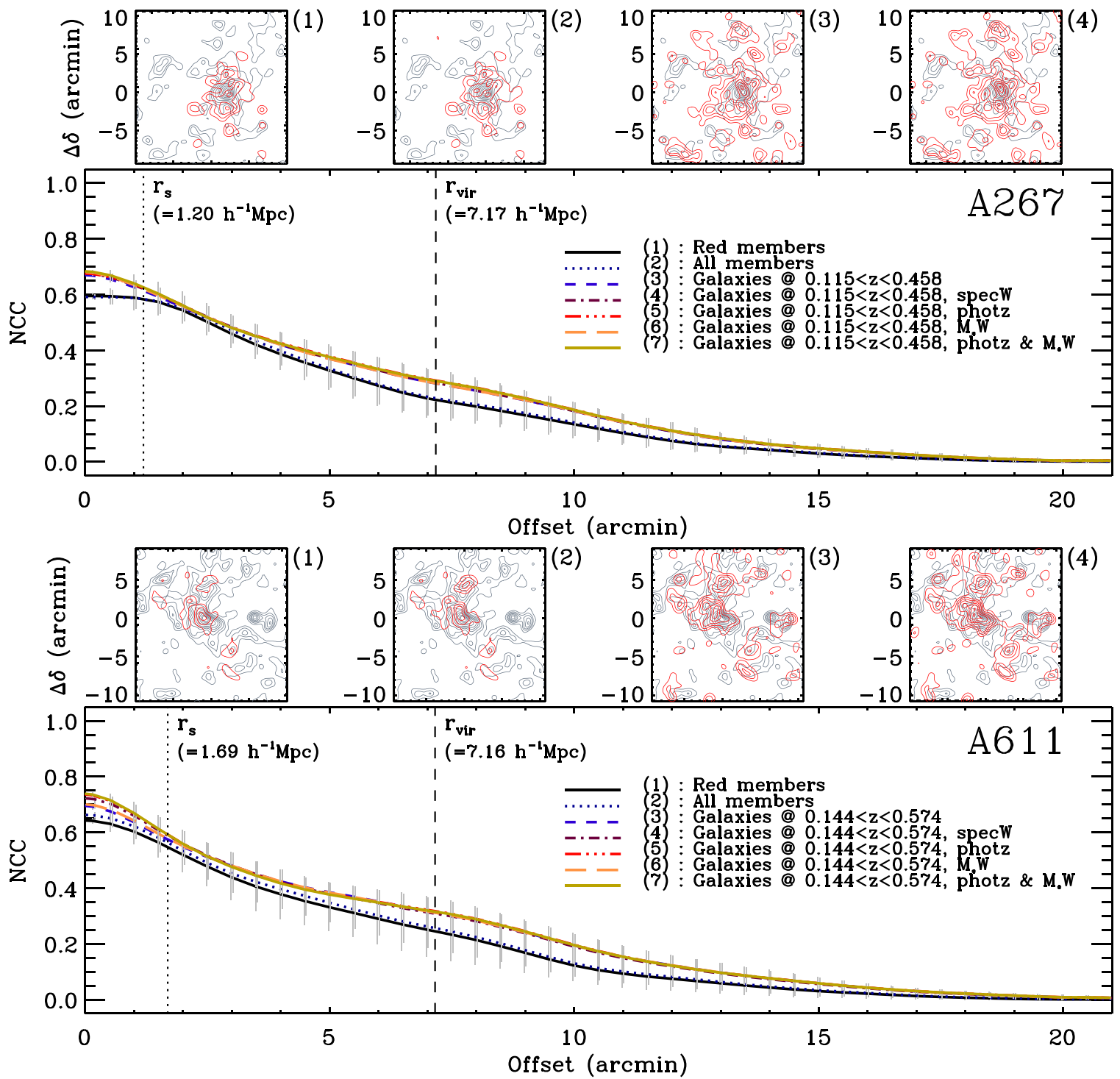

Figure 8. Same as Figure 7 (except for the 2D NCC map), but for A267 and A611. Gray and red contours indicate weak-lensing and galaxy number density maps, respectively.

(A color version of this figure is available in the online journal.)

background galaxies can affect the apparent ellipticity of cluster mass distribution in a weak-lensing map. The mass profile of this cluster derived from the two-dimensional weak-lensing map is correspondingly very noisy (see Figure 29 in Okabe et al. 2010). The noisy mass profile may result from the complex contribution of foreground and background galaxies to cluster shape.

A689. The X-ray emission of this cluster is dominated by a central BL Lac object (Giles et al. 2012); thus, an X-ray luminosity of this cluster is smaller than the other clusters in the sample (see Rines et al. 2013 for details). The weak-lensing map for this cluster shows several significant peaks. The galaxy number density maps based on members alone also show peaks in the central and upper regions of the map. The peaks in the lower regions of the map appear significantly enhanced when we include foreground and background galaxies. The crosscorrelation signal when we include foreground and background galaxies is systematically larger than the one based on members only. In fact, Okabe et al. (2010) could not derive the mass profile of this cluster because the projected mass distribution in the weak-lensing map is so complex. The difficulty in deriving the cluster mass profile occurs in part because the cluster itself is complex and the contribution of foreground and background galaxies further complicates the weak-lensing signal.

A697. The spectroscopic completeness for this cluster within the weak-lensing map is one of the highest (89\%) in our sample. The cluster galaxies are centrally concentrated. They dominate the signal in the galaxy number density map even when we include foreground and background galaxies. Thus, the radial profiles of the correlation signal for several subsamples do not differ significantly. Okabe et al. (2010) could not fit the mass profile of this cluster with a singular isothermal sphere (SIS) model but could fit it with either a cored isothermal sphere (CIS) or NFW model.

A750. This cluster is the closest cluster $(z=0.164)$ in the sample. The difference in correlation signal at zero offset between cases based on members alone and including foreground/ background galaxies is the largest in the sample. This result occurs because there are two clusters with similar redshifts aligned nearly along the line of sight in this field: $z=0.164$ for $\mathrm{A} 750$ and $z=0.1767$ for MS $0906.5+1110$ (Carlberg et al. 


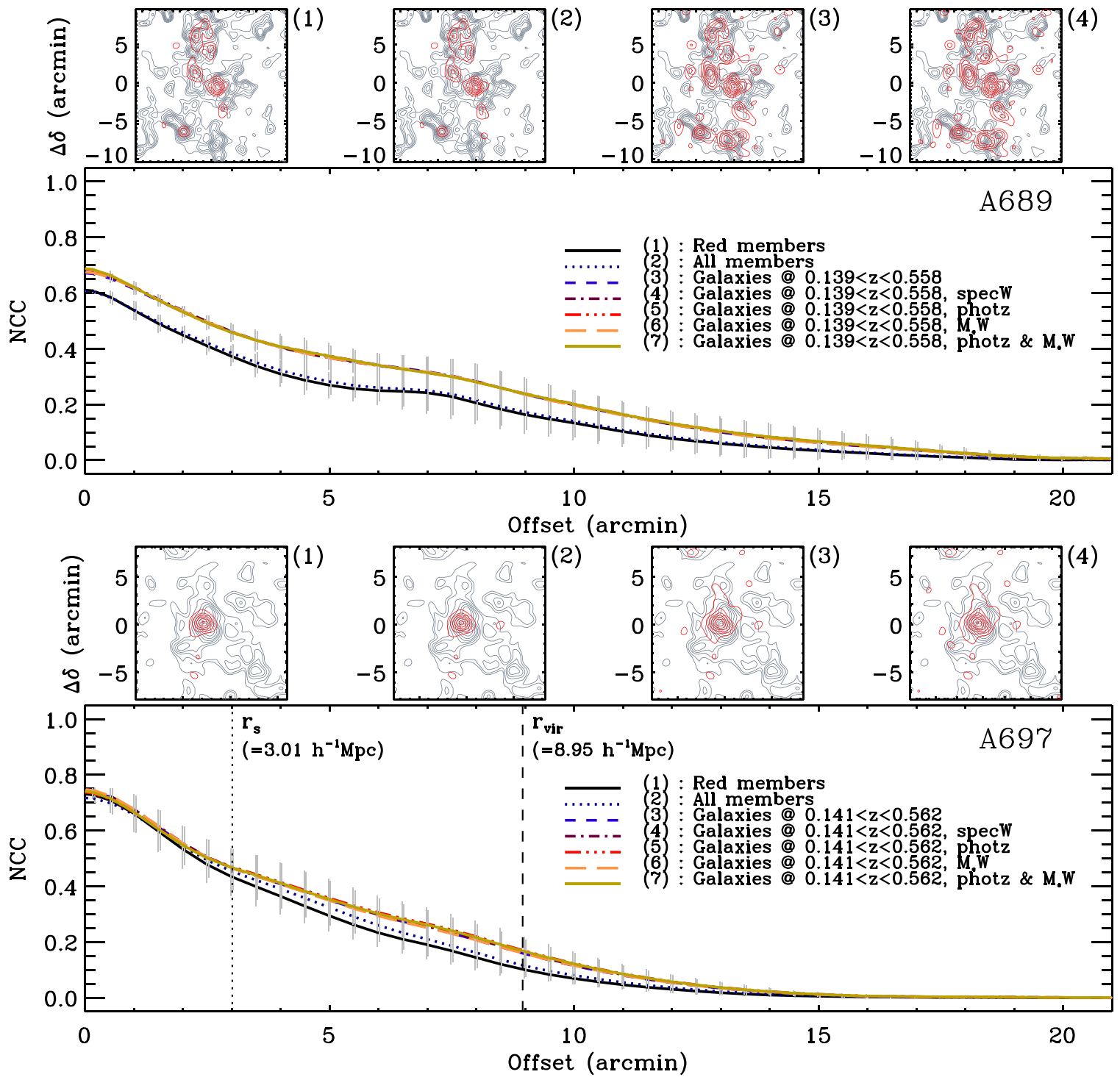

Figure 9. Same as Figure 7 (except for the 2D NCC map), but for A689 and A697. Gray and red contours indicate weak-lensing and galaxy number density maps, respectively.

(A color version of this figure is available in the online journal.)

1996; Rines et al. 2013; Geller et al. 2013). MS 0906.5+1110 is more X-ray luminous than A750. Two clusters are often used without a clear distinction between the two. Their velocity difference is $3250 \mathrm{~km} \mathrm{~s}^{-1}$ in the cluster rest frame. Our redshift survey resolves these two. The galaxies in MS 0906.5+1110 are not selected as A750 members, but they contribute significantly to the lensing signal (see Section 5 of Geller et al. 2013 for details). Therefore, the mass based on weak lensing is roughly double the true cluster mass (Geller et al. 2013). In fact, Okabe et al. (2010) could not derive a mass profile of this cluster because the mass distribution in the weak-lensing map is so complex.

A963. At small offsets, the correlation signal when we include photometric redshifts for foreground and background galaxies are slightly smaller than the one based on members only. The large dispersion in the number density map based on foreground and background galaxies with photometric redshifts affects the normalization of the correlation signal (see Equation (1)). Considering the small difference and the large uncertainty in photometric redshifts, the effect is not statistically significant.
Interestingly, Okabe et al. (2010) could not obtain an acceptable fit to the mass profile of this cluster with any of the three models (NFW, SIS, and CIS). This cluster is an exceptional case where weak lensing does not provide a robust mass profile even though the radial profiles of the cross-correlation signal for several subsamples do not differ significantly.

$R X J 1720.1+2638$. The overall spectroscopic completeness for this cluster in the weak-lensing map is one of the highest (89\%) in the sample. The Chandra X-ray observations show cold fronts in this cluster (Mazzotta et al. 2001), probably resulting from sloshing induced by the gravitational perturbation through minor mergers with small groups (Owers et al. 2011). A secondary peak to the north of the main concentration in the weak-lensing map appears as an overdensity in the number density map based on cluster members alone. The onedimensional cross-correlation signals are similar to one another, suggesting that the contribution of foreground and background galaxies to the weak-lensing map is not significant in this cluster field. Okabe et al. (2010) found a good fit to their models for a weak-lensing mass profile for this cluster. 


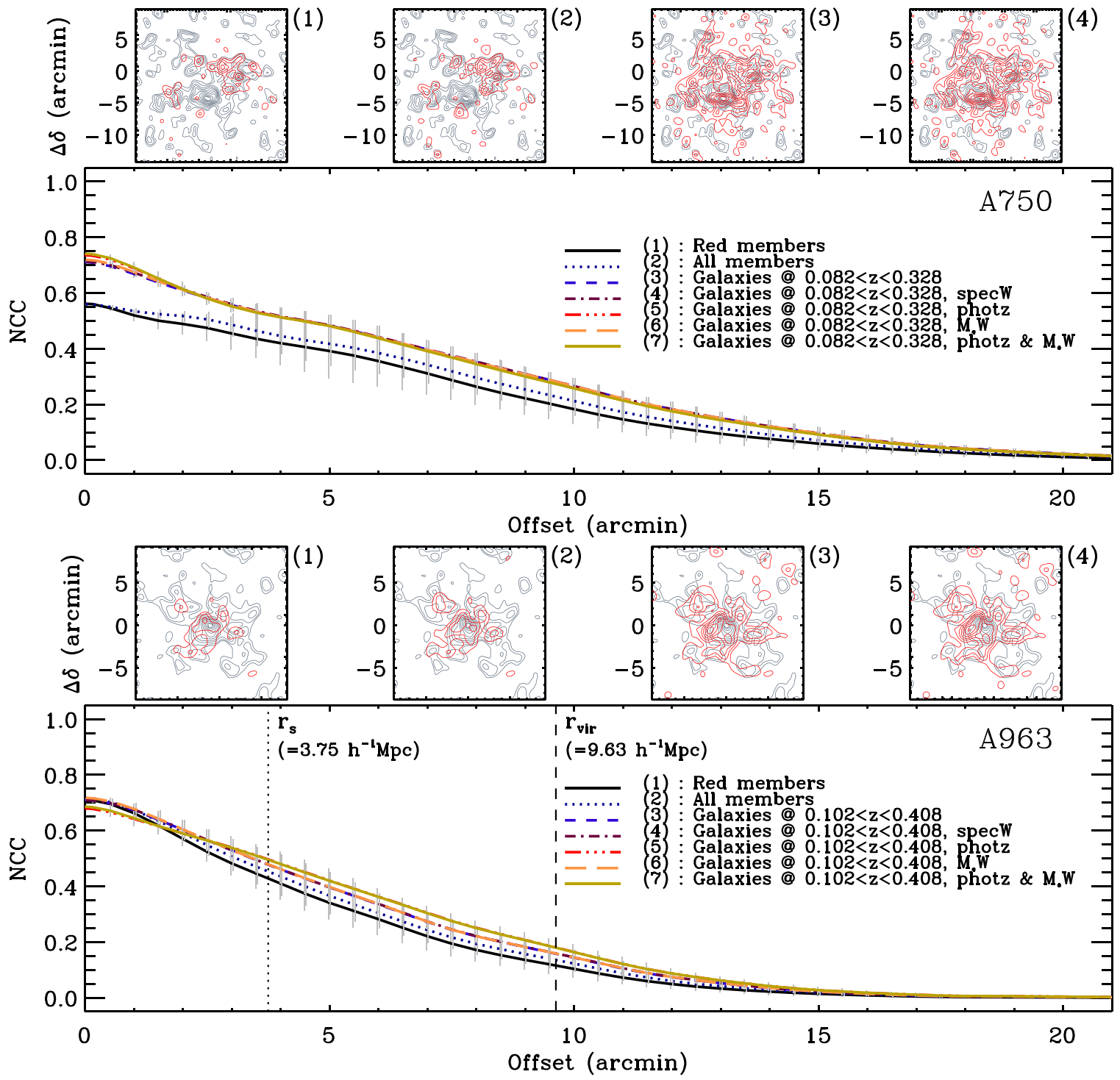

Figure 10. Same as Figure 7 (except for the 2D NCC map), but for A750 and A963. Gray and red contours indicate weak-lensing and galaxy number density maps, respectively.

(A color version of this figure is available in the online journal.)

RXJ2129.6+0005. This cluster has the smallest number of galaxies with spectroscopic redshifts in the weak-lensing map (N: 156). The secondary peaks to the west and to the southsouthwest of the main concentration appear only when we include foreground and background galaxies, but the amplitudes of the peaks are low. Therefore, the one-dimensional correlation signal remains similar. Okabe et al. (2010) found a robust fit to the weak-lensing mass profile for this cluster.

Comparison of the cross-correlation results with the weaklensing mass profiles in Okabe et al. (2010) for the nine clusters yields diverse results. There are three clusters (A689, A750, and A383) where the normalized cross-correlation signal including foreground and background galaxies significantly exceeds the signal based on cluster members alone; Okabe et al. (2010) could not derive a stable mass profile for two clusters (e.g., A689 and A750) because of the complex projected mass distribution in the weak-lensing map and could not obtain an acceptable fit to the mass profile of A383 with an NFW, SIS, or CIS model. For two clusters where the normalized cross-correlation signal including foreground and background galaxies slightly exceeds the signal based on cluster members alone at zero offset (e.g., A267, A611), Okabe et al. (2010) obtained a robust weak-lensing mass profile. Okabe et al. (2010) derived a stable weak-lensing mass profile for two clusters where there is no significant difference between the normalized cross-correlation signal for several galaxy subsamples (e.g., RX J1720.1+2638, RX J2129.6+0005). Okabe et al. (2010) could not obtain an acceptable model fit to the derived mass profiles of two clusters (e.g., A697, A963) even though there is no significant difference in the normalized cross-correlation signal for the galaxy subsamples we investigate here. Although the nine clusters show diverse results, a normalized cross-correlation signal including foreground and background galaxies that significantly exceeds the signal based on cluster members alone appears to be a good proxy for an underlying systematic problem in the interpretation of the projected mass distribution as revealed by the weaklensing map. Problems may result from resolved substructure within the cluster (e.g., A689) and/or from the contribution of large-scale structure superimposed along the line of sight (e.g., A383, A750). 


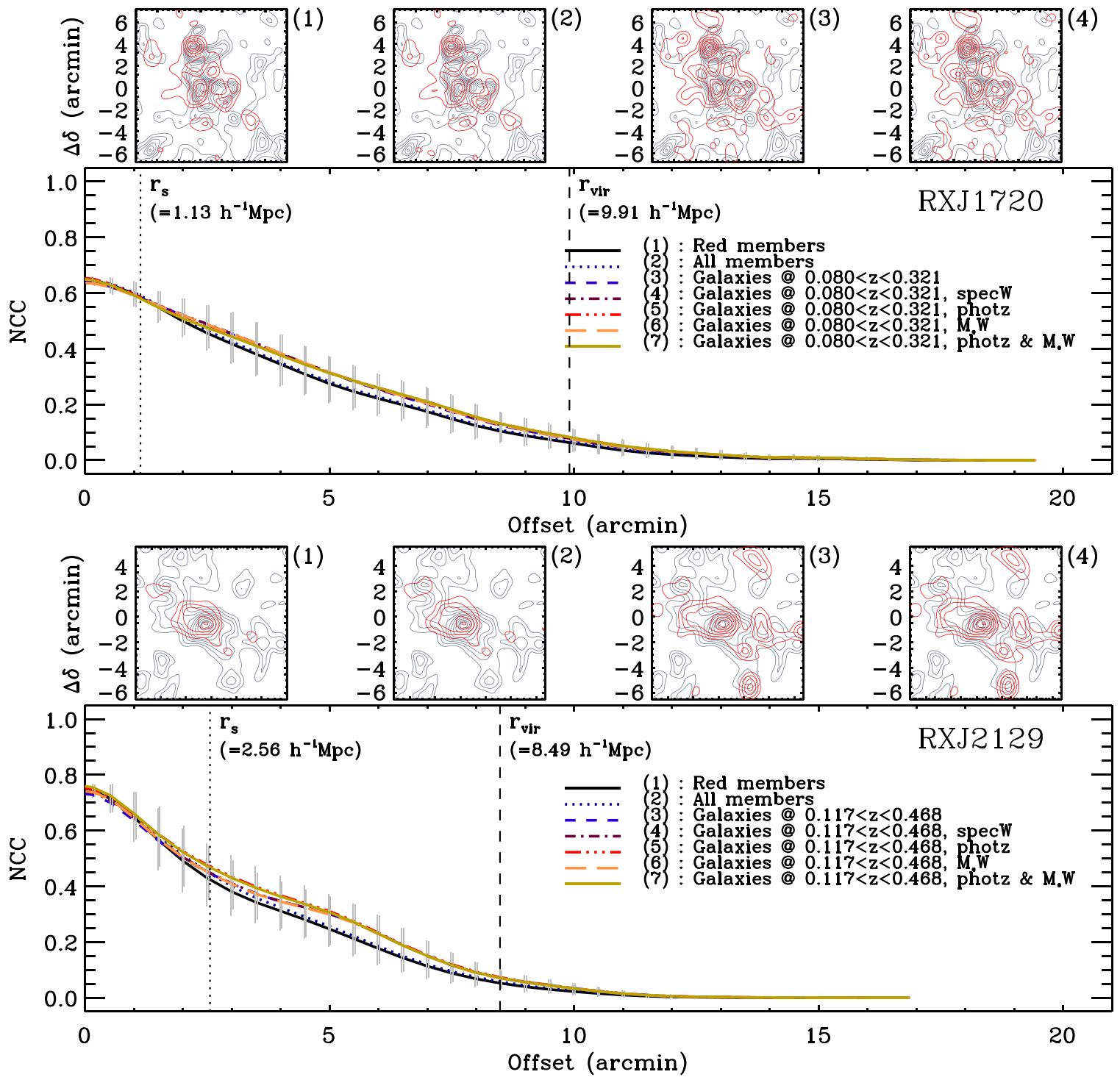

Figure 11. Same as Figure 7 (except for the 2D NCC map), but for RX J1720.1+2638 and RX J2129.6+0005. Gray and red contours indicate weak-lensing and galaxy number density maps, respectively.

(A color version of this figure is available in the online journal.)

\section{DISCUSSION}

We use dense redshift surveys in the fields of nine $z \sim 0.2$ galaxy clusters to cross-correlate galaxy number density maps with weak-lensing maps. The cross-correlation signal when we include foreground and background galaxies exceeds the one based on cluster members alone (see Figures 7-11); the crosscorrelation for the full sample is not negligible even outside the cluster virial radii.

We summarize the results of the cross-correlation for the nine clusters in Figure 12. We plot the difference in the normalized correlation signal between the cases including all the galaxies around clusters and based on cluster members alone (i.e., cases 7 and 2 in Figure 7) in the top panel. The bottom panel shows the cumulative $(<R)$, fractional excess for the case including all the galaxies around the cluster relative to the case based on cluster members alone (i.e., cases 7 and 2 in Figure 7).

The bottom panel shows that the cumulative fractional excess changes with offset and increases up to $30 \%$. The typical statistical error in the cumulative fractional excess is $3 \%-5 \%$.
The nine clusters show different patterns, reflecting different large-scale structure along the line of sight. At the typical virial radius of our cluster samples (i.e., $\sim 1.3 h^{-1} \mathrm{Mpc}$ ), the cumulative fractional excess is $5 \%-23 \%$. These excesses are roughly consistent with the results based on simulation data (Hoekstra et al. 2011b); uncorrelated large-scale structure along the line of sight contributes to an uncertainty in weak-lensing cluster mass of $10 \%-25 \%$ for $z \sim 0.2$ clusters (see also Hoekstra 2001 for analytical prediction). The increase of the cumulative fractional excess with offset is also consistent with the idea that the weak-lensing analysis tends to overestimate cluster masses in the outer regions (e.g., see Figure 13 in Geller et al. 2013). Studies of much larger cluster samples suggest that one can determine robust 3D mass profiles of galaxy clusters from weak-lensing measurements based on the ensemble; a halo model approach to analysis of the ensemble can treat the correlated structure (Johnston et al. 2007a, 2007b; Leauthaud et al. 2010).

One interesting aspect of Figure 12 is that the fractional excess is significant even at zero offset for some clusters (e.g., A267, 


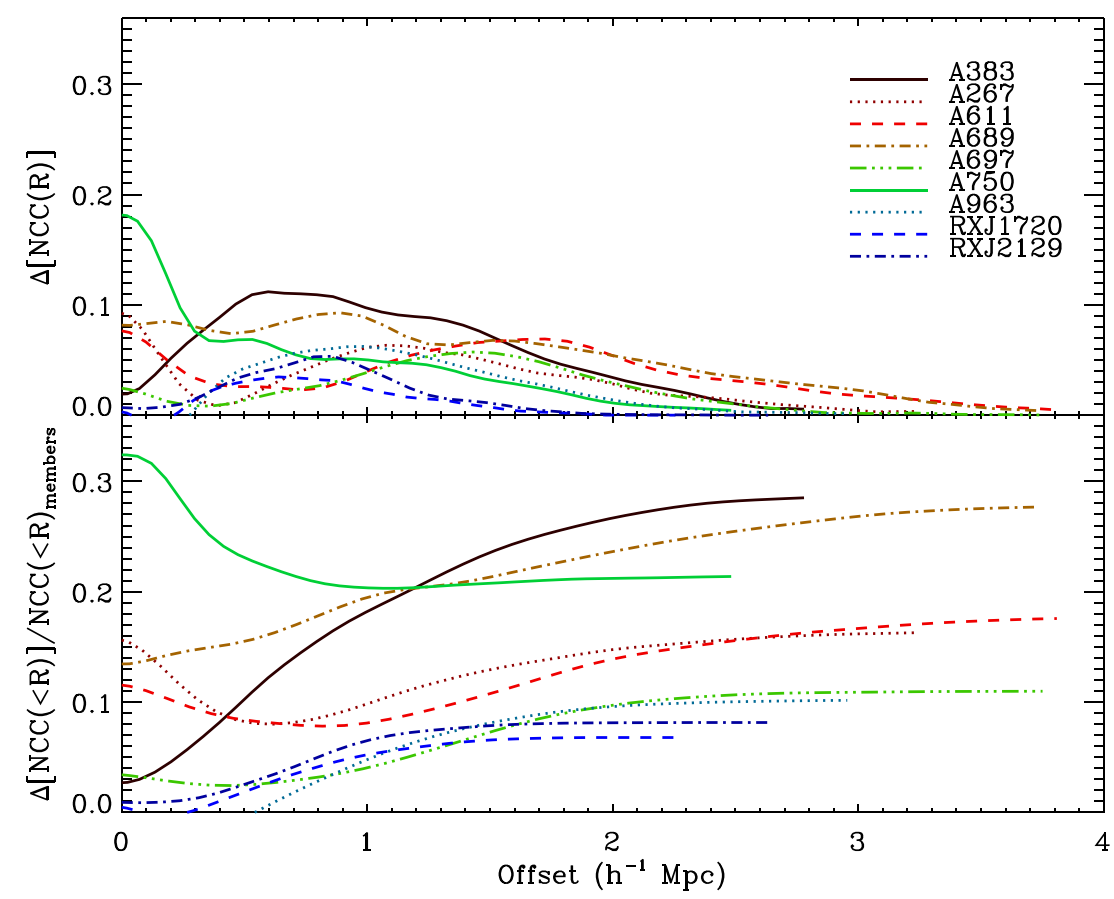

Figure 12. Top: difference in normalized correlation signal for cases with cluster member galaxies (i.e., case 2 in Figure 7 ) and with all the galaxies in the redshift range $0.5 z_{\mathrm{cl}}<z<2 z_{\mathrm{cl}}$, complemented by photometric redshifts and weighed by stellar masses (i.e., case 7 in Figure 7 ) as a function of offset for nine clusters in this study. Bottom: same as the top panel, but for the cumulative fractional difference in a normalized correlation signal.

(A color version of this figure is available in the online journal.)

A611, A689, and A750). Thus, we might expect a significant number of galaxies inside the Subaru FOV that are not cluster members but that contribute to the lensing signal. Figure 13 shows a redshift histogram for the galaxies in the weak-lensing map of each cluster. The hatched and open histograms show the cluster members and all the galaxies with spectroscopic/ photometric redshifts at $m_{\mathrm{r}, \text { Petro, } 0} \leqslant 20.5$, respectively. The histogram clearly shows that the fields of the clusters with large fractional excess have non-member galaxies close to the mean cluster redshift.

Based on simulations, Becker \& Kravtsov (2011) emphasize that large-scale structure associated (or correlated) with individual clusters makes a non-negligible contribution $(\sim 20 \%)$ to the scatter of weak-lensing mass estimates in addition to the contribution of uncorrelated large-scale structure along the line of sight (10\%-25\%; Hoekstra et al. 2011b). We highlight the field of A750 in Section 3.2.2 where the contribution of the lensing signal from a nearby superimposed cluster is about a factor of two.

The vertical dashed lines in Figure 13 indicate the redshift range that we use to include foreground and background galaxies when we make the galaxy number density map for each cluster (i.e., $0.5 z_{\mathrm{cl}}$ and $2 z_{\mathrm{cl}}$ ). The nine clusters are at similar redshifts (i.e., $z \sim 0.2$ ); thus, the redshift ranges for including foreground and background galaxies are also similar. Moreover, examination of this plot also shows that the amplitude of the cumulative fractional excess in the bottom panel of Figure 12 does not depend on the cluster redshift, suggesting that the small difference in cluster redshifts in our sample does not introduce a bias.

The spectroscopic completeness of our redshift survey for the nine clusters is very high $\left(70 \%-89 \%\right.$ at $\left.m_{\mathrm{r}, \mathrm{Petro}, 0} \leqslant 20.5\right)$. However, our redshift surveys are not deep enough to include a large number of galaxies at the high-redshift end of the lensing kernel (e.g., galaxies fainter than $m_{\mathrm{r}, \text { Petro }, 0}=20.5$ at $0.1 \lesssim z \lesssim 0.4$ for $z \sim 0.2$ cluster). Thus, our estimates of the contribution of large-scale structure along the line of sight to the cluster lensing maps could be lower limits; the real contribution could be larger even though photometric redshifts mitigate this issue to some extent.

Okabe et al. (2010) studied the mass profiles of the nine clusters derived from the two-dimensional weak-lensing maps in this study. They used three mass models to fit the derived mass profiles: NFW, SIS, and CIS. Interestingly, they could not derive the mass profiles of A750 and A689 because the mass distribution in the weak-lensing maps is complex in these systems. They could fit the mass profiles with their three models for most clusters but could not obtain an acceptable fit to the mass profile of A383 with any model. These three clusters (A383, A689, and A750) show the largest fractional excesses $(11 \%-30 \%)$ in the integrated normalized correlation signal at offset $>0.5 h^{-1} \mathrm{Mpc}$. The range of possible impacts of the superimposed structure on cluster mass estimates is large: a few percent for A383 (Geller et al. 2014a) and up to a factor of two for A750 (Geller et al. 2013). These results suggest that the excess in the integrated cross-correlation signal could be a useful proxy for assessing the reliability of weak-lensing cluster mass estimates.

We cross-correlate two independent measurements, the galaxy number density map, and the lensing convergence field, $\kappa$, for each cluster. As suggested by Van Waerbeke et al. (2013), it would be interesting to use the galaxy number density map to reconstruct a model convergence map for comparison with the observed one. However, this procedure requires knowledge of positions and redshifts of the source galaxies that are not currently available. 


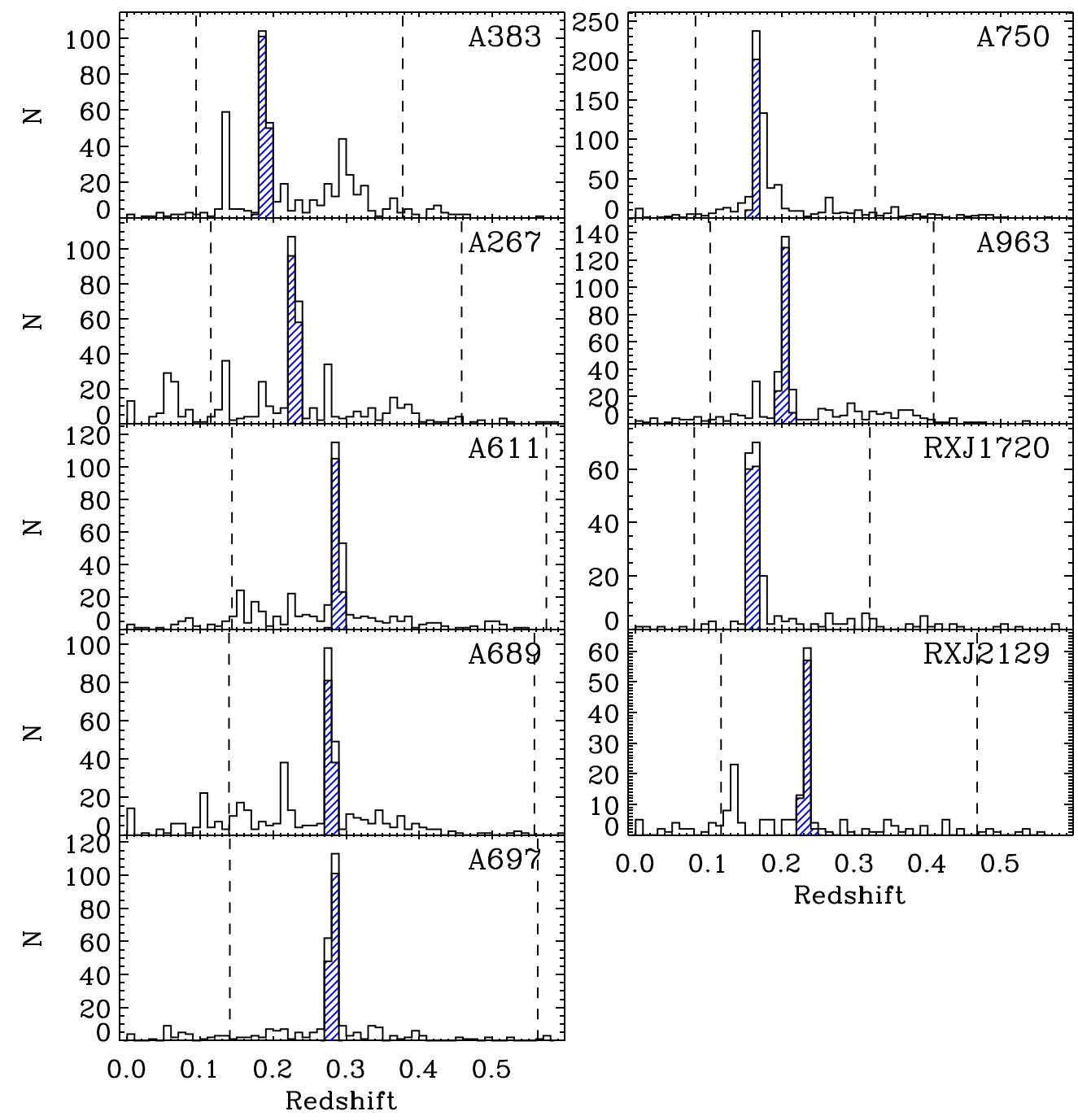

Figure 13. Redshift histograms for the galaxies in the Subaru FOVs of galaxy clusters. The blue hatched histogram shows the cluster members. Open histograms show all the galaxies with spectroscopic or photometric redshifts at $m_{\mathrm{r}, \text { Petro, } 0}<20.5$. Two vertical dashed lines indicate the redshift range when we include foreground and background galaxies (i.e., $0.5 z_{\mathrm{cl}}$ and $2 z_{\mathrm{cl}}$ ).

(A color version of this figure is available in the online journal.)

\section{CONCLUSIONS}

We use dense redshift surveys in the fields of nine $z \sim 0.2$ galaxy clusters to compare the structures identified in weaklensing and galaxy number density maps. We combine 2087 new MMT/Hectospec redshifts and the data in the literature to make the overall spectroscopic completeness in the weaklensing maps high (70\%-89\%) and uniform. With these dense redshift surveys, we first construct galaxy number density maps using several galaxy subsamples. Our primary results are as follows.

1. The global morphology of the spatial distribution of cluster members alone is similar to the shape of the cluster peak in weak-lensing maps. However, in some clusters (e.g., A611), the apparent shape of the cluster peak in the weak-lensing map may be affected by the contribution of foreground and background galaxies.

2. The red cluster galaxies dominate the cluster weak-lensing signal, and blue cluster galaxies contribute little to the number density maps of cluster members. These results suggest that the red populations are reliable tracers of the mass distribution of galaxy clusters provided that the selection is dense and broad enough around the red sequence. This result supports the approach often taken in strong-lensing analysis (Broadhurst et al. 2005; Zitrin et al. 2009; Medezinski et al. 2010).

3 . The correspondence between the galaxy number density and weak-lensing maps is even more remarkable when we include foreground and background galaxies in the galaxy number density maps, reflecting the contribution of superimposed large-scale structure along the line of sight to the $\kappa$ map.

We cross-correlate the galaxy number density maps with the weak-lensing maps, and we find the following.

1. The cross-correlation signal when we include foreground and background galaxies at $0.5 z_{\mathrm{cl}}<z<2 z_{\mathrm{cl}}$ is always larger than for the case with cluster members alone. The fractional excess of the integrated normalized correlation signal for the case including foreground and background galaxies relative to the case based on cluster members alone is $10 \%-23 \%$ at the cluster virial radius. This excess can be as high as $30 \%$ depending on the cluster. 

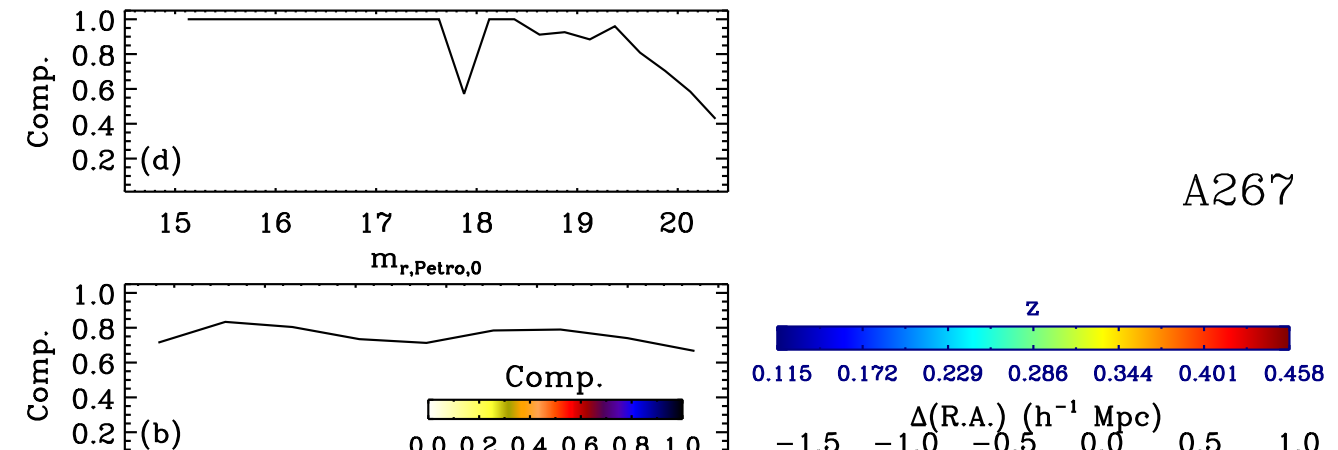

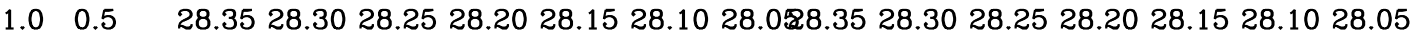
Comp R.A. (deg)

R.A. (deg)
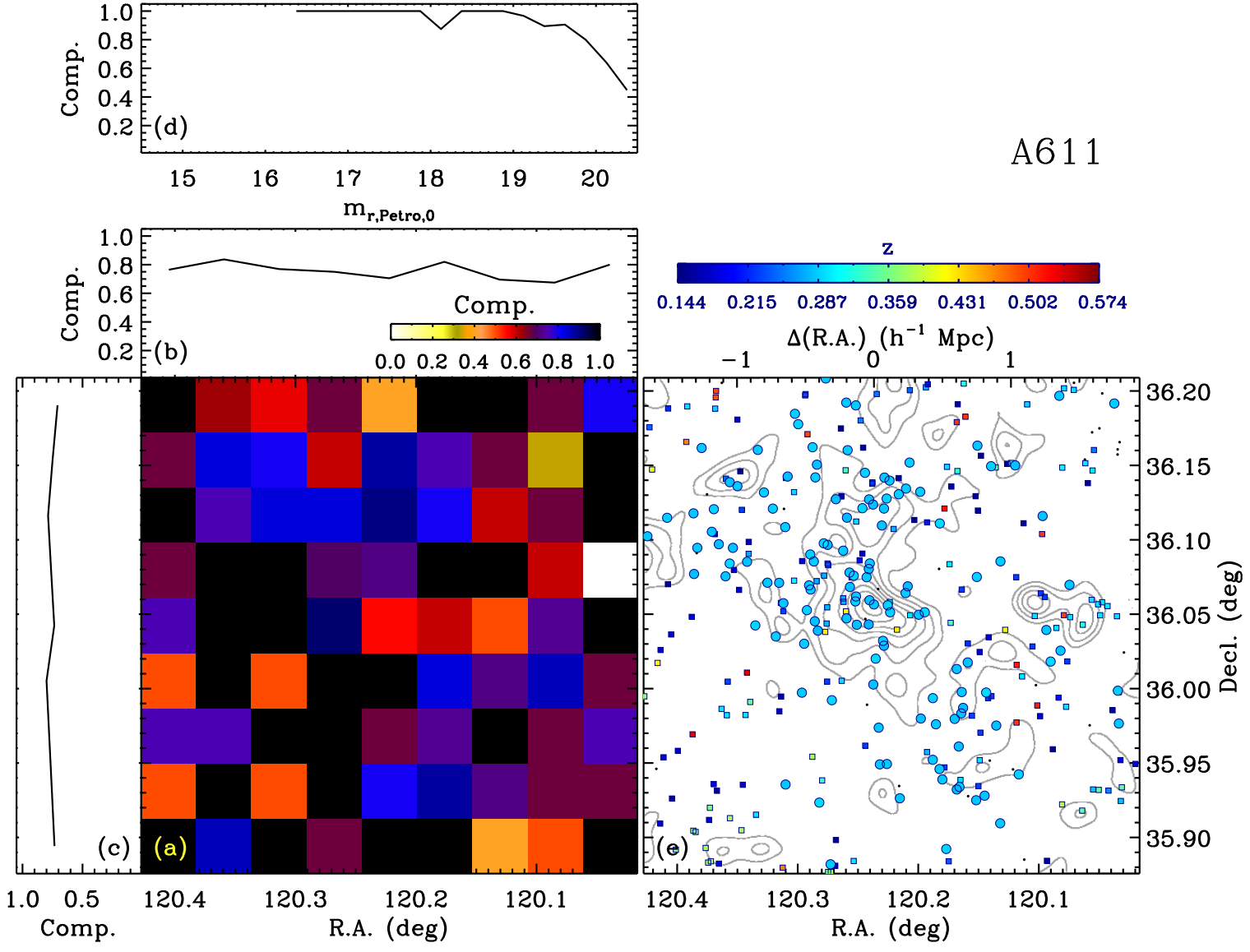

Figure 14. Same as Figure 4, but for A267 and A611.

(A color version of this figure is available in the online journal.) 


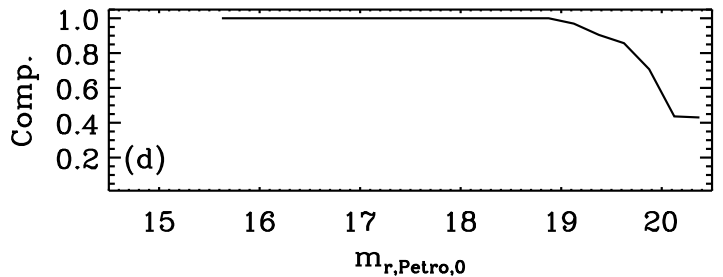

\section{A689}
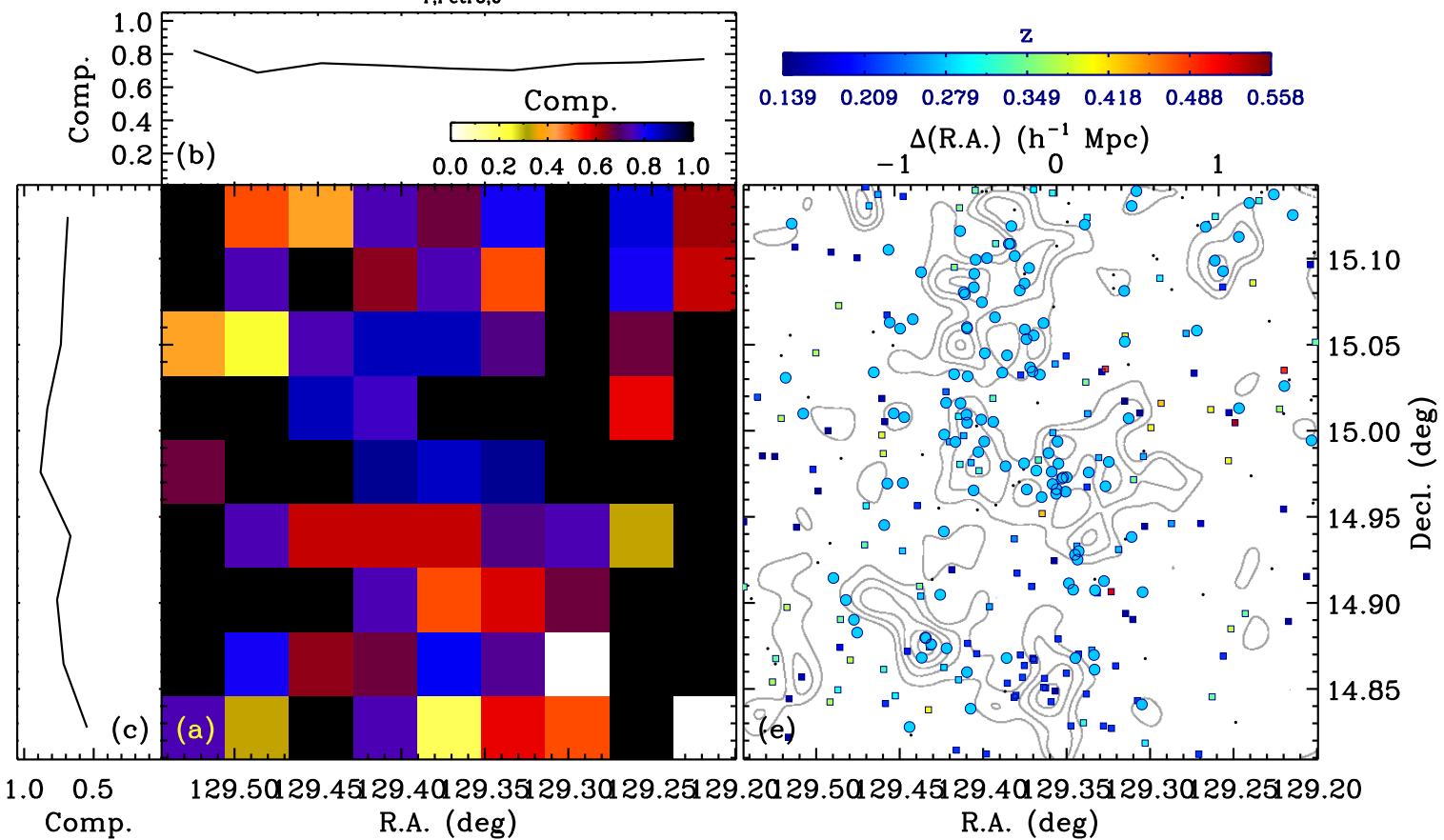

$\begin{array}{lll}1.0 & 0.5 & 129.5 \alpha 29.45129 .4 \alpha 29.35129 .3 \alpha 29.25129 .2 \alpha 29.5 a 29.45129 .4 \alpha 29.35129 .3 \alpha 29.25129 .20\end{array}$
Comp.
R.A. (deg)
R.A. (deg)
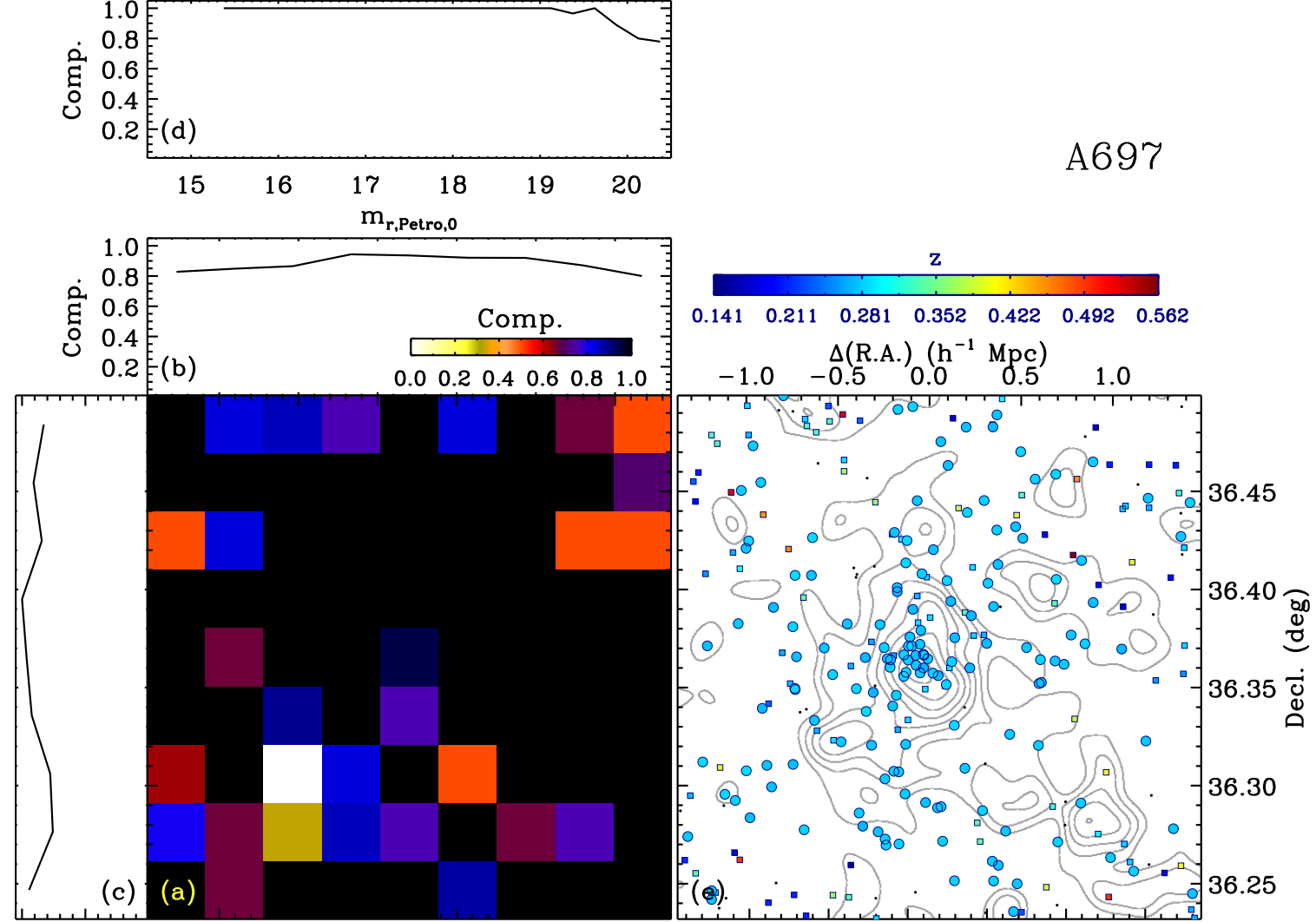

$1.0 \quad 0.5$

130.85130 .80130 .75130 .70130 .65130 .60 Comp.

R.A. (deg)

130.85130 .80130 .75130 .70130 .65130 .60

Figure 15. Same as Figure 4, but for A689 and A697.

(A color version of this figure is available in the online journal.) 


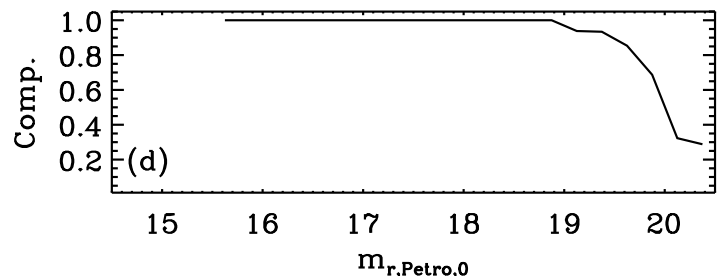

A750
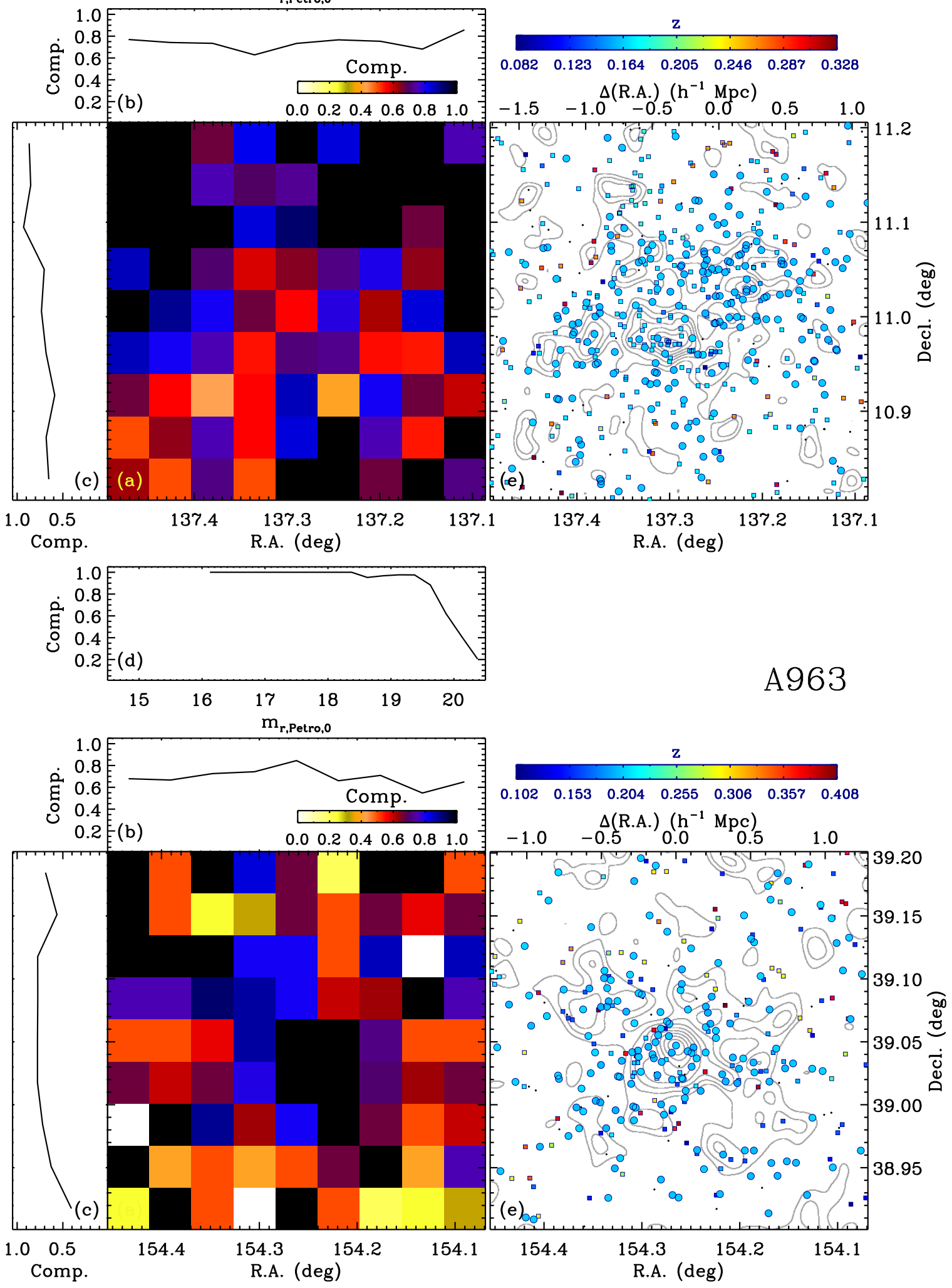

Figure 16. Same as Figure 4, but for A750 and A963.

(A color version of this figure is available in the online journal.) 


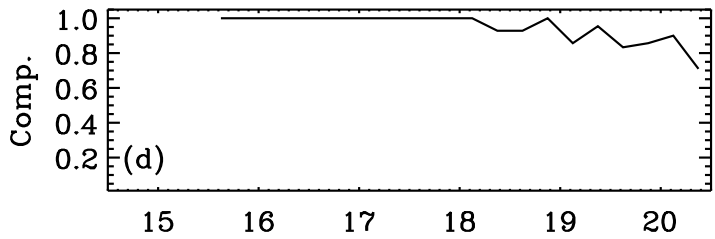

\section{RXJ 1720}
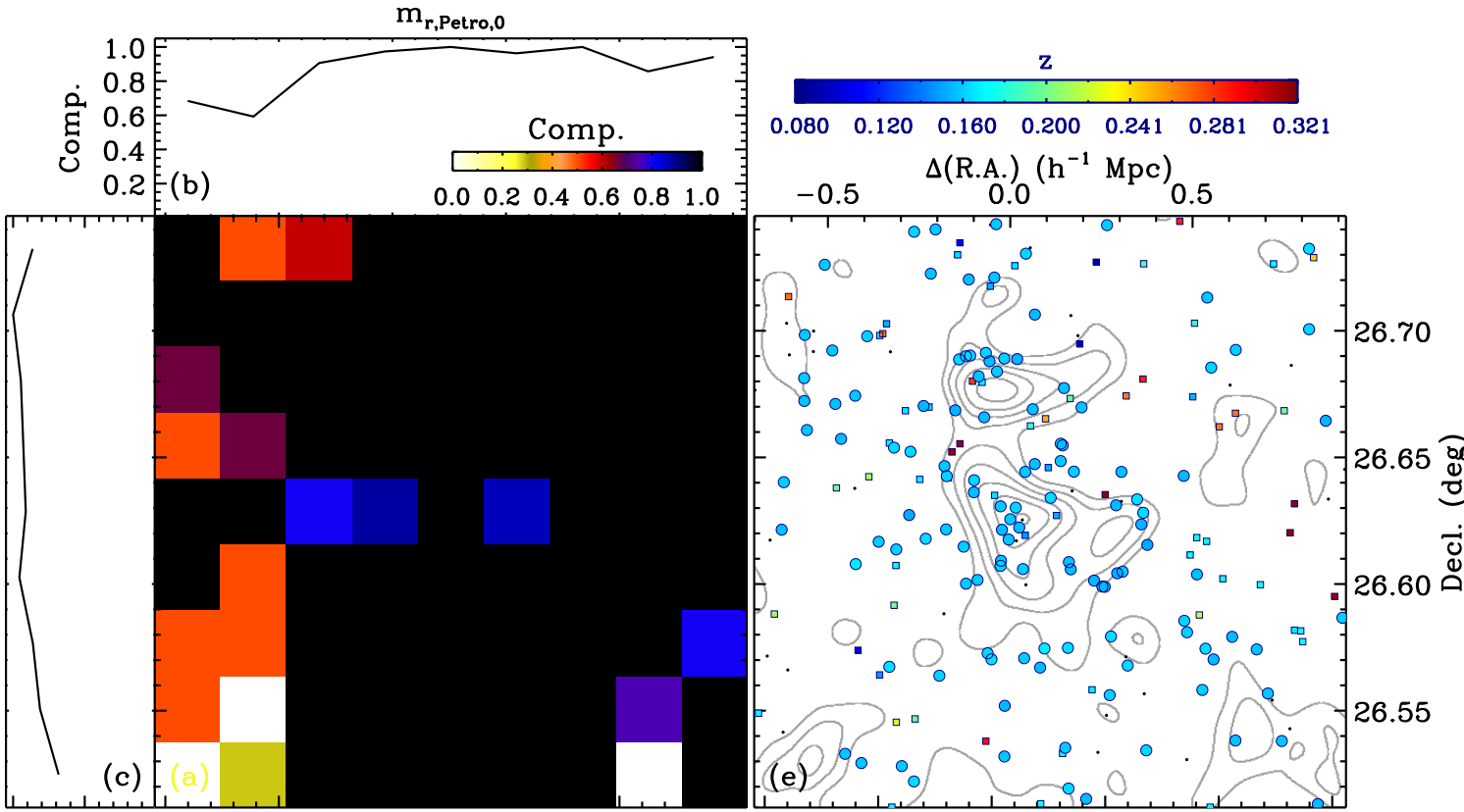

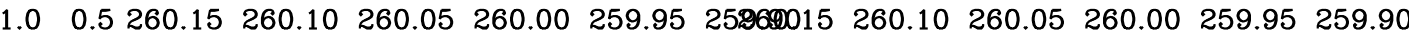
Comp.

R.A. (deg)

R.A. (deg)

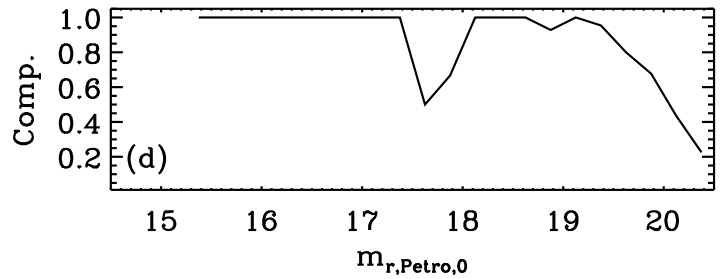

\section{RXJ2129}

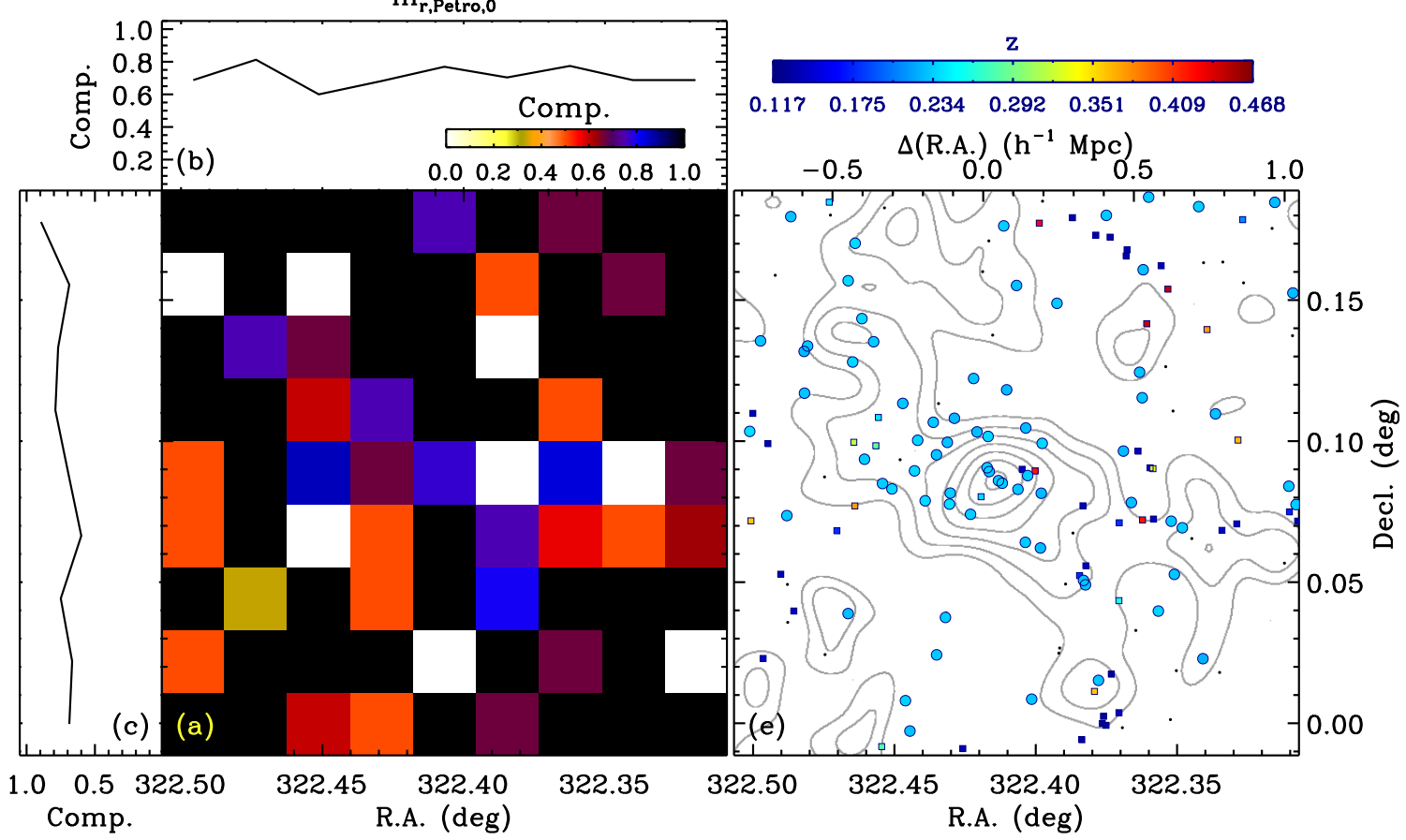

Figure 17. Same as Figure 4, but for RX J1720.1+2638 and RX J2129.6+0005.

(A color version of this figure is available in the online journal.) 


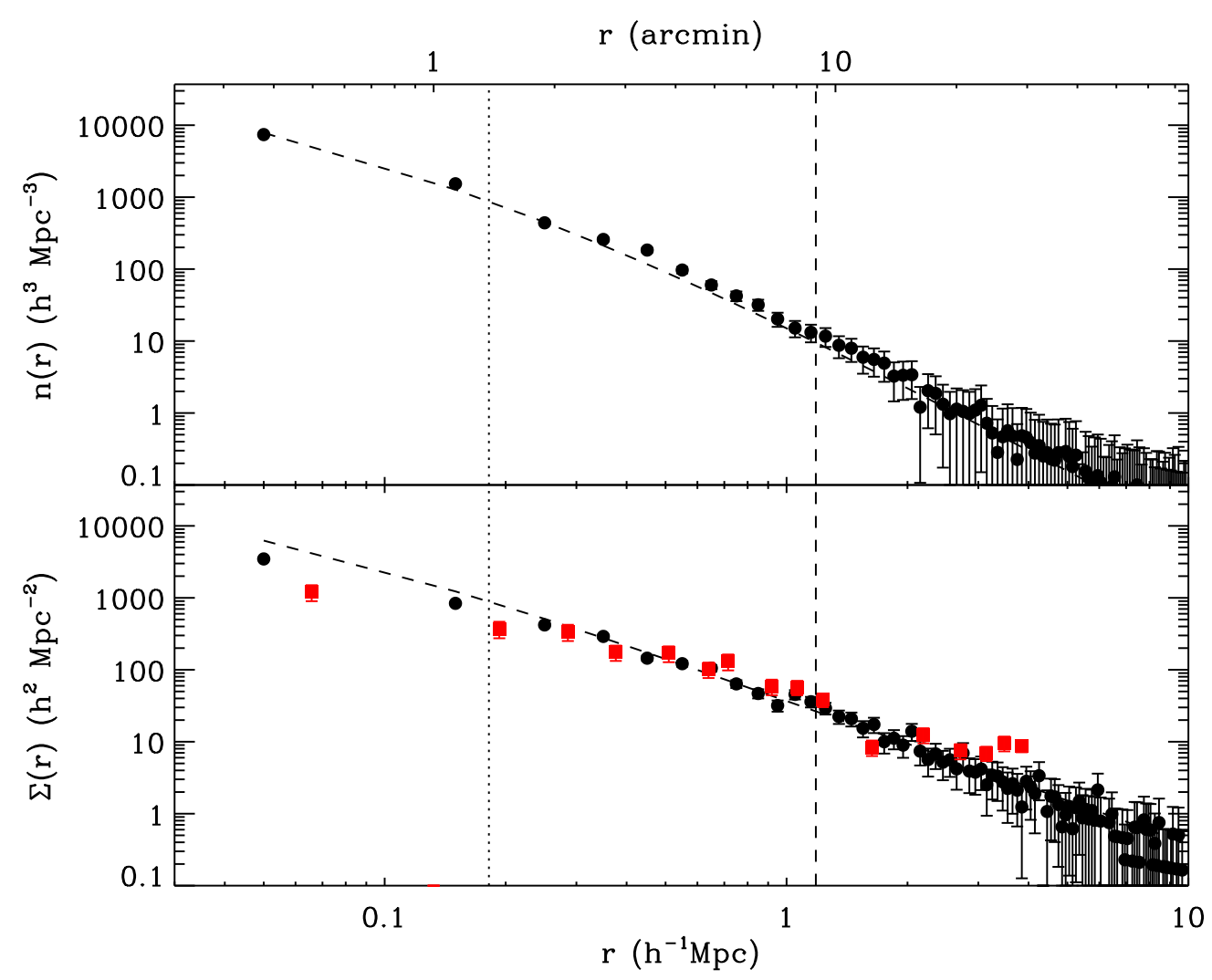

Figure 18. Top: galaxy number density profile for a simulated cluster (filled circles). Dashed line is the input NFW profile with $r_{200}=1.184\left(h^{-1} \mathrm{Mpc}\right)$ and $c_{200}=6.51$ (Newman et al. 2013). Vertical dotted and dashed lines indicate $r_{s}$ and $r_{200}$, respectively. Bottom: same as the top panel, but for projected galaxy number density profile. Red squares are observed galaxy number density profile of A383 after completeness correction derived in this study.

(A color version of this figure is available in the online journal.)

2. Superimposed structure close to the cluster in redshift space contributes to the weak-lensing peaks more significantly than unrelated large-scale structure along the line of sight.

3. The mass profiles of three clusters (A383, A689, and A750) with the largest fractional excesses (11\%-30\%) of the integrated normalized correlation signal are also not well constrained in weak lensing (Okabe et al. 2010). Thus, the excess in the integrated normalized correlation signal could be a useful proxy for assessing the reliability of weaklensing cluster mass estimates.

A dense redshift survey of galaxy clusters is important for understanding the meaning of a weak-lensing $\kappa$ map. We plan to extend this study to a larger cluster sample including merging clusters in a forthcoming paper (H. S. Hwang et al., in preparation).

Future exploration with deep spectroscopic or even photometric redshift surveys covering all the galaxies including faint ones in the lensing kernel is important for refining the estimates of the contribution of large-scale structure along the line of sight accurately. To resolve the structure near clusters that contribute to weak-lensing maps significantly, a spectroscopic survey is crucial. Techniques that treat the redshift survey simultaneously with weak lensing may eventually provide very powerful probes of mass distribution uncontaminated by superimposed structure along the line of sight.

We thank the anonymous referee for helpful comments. We thank Perry Berlind and Michael Calkins for operating the Hectospec and Susan Tokarz and Sean Moran for reducing the Hectospec data. We also thank Dan Coe, Ian Dell' Antonio, Nobuhiro
Okabe, Graham Smith, Keiichi Umetsu, and Adi Zitrin for helpful comments in early stages of this work. The Smithsonian Institution supports the research of M.J.G. and H.S.H. A.D. acknowledges partial support from the INFN grant Indark and from the grant Progetti di Ateneo/CSP TO_Call2_2012_0011 "Marco Polo" of the University of Torino. Funding for SDSSIII has been provided by the Alfred P. Sloan Foundation, the Participating Institutions, the National Science Foundation, and the U.S. Department of Energy Office of Science. The SDSS-III website is http://www.sdss3.org/. SDSS-III is managed by the Astrophysical Research Consortium for the Participating Institutions of the SDSS-III Collaboration including the University of Arizona, the Brazilian Participation Group, Brookhaven National Laboratory, Carnegie Mellon University, University of Florida, the French Participation Group, the German Participation Group, Harvard University, the Instituto de Astrofisica de Canarias, the Michigan State/Notre Dame/JINA Participation Group, Johns Hopkins University, Lawrence Berkeley National Laboratory, Max Planck Institute for Astrophysics, Max Planck Institute for Extraterrestrial Physics, New Mexico State University, New York University, Ohio State University, Pennsylvania State University, University of Portsmouth, Princeton University, the Spanish Participation Group, University of Tokyo, University of Utah, Vanderbilt University, University of Virginia, University of Washington, and Yale University. This research has made use of the NASA/IPAC Extragalactic Database (NED), which is operated by the Jet Propulsion Laboratory, California Institute of Technology, under contract with the National Aeronautics and Space Administration.

Facility: MMT 

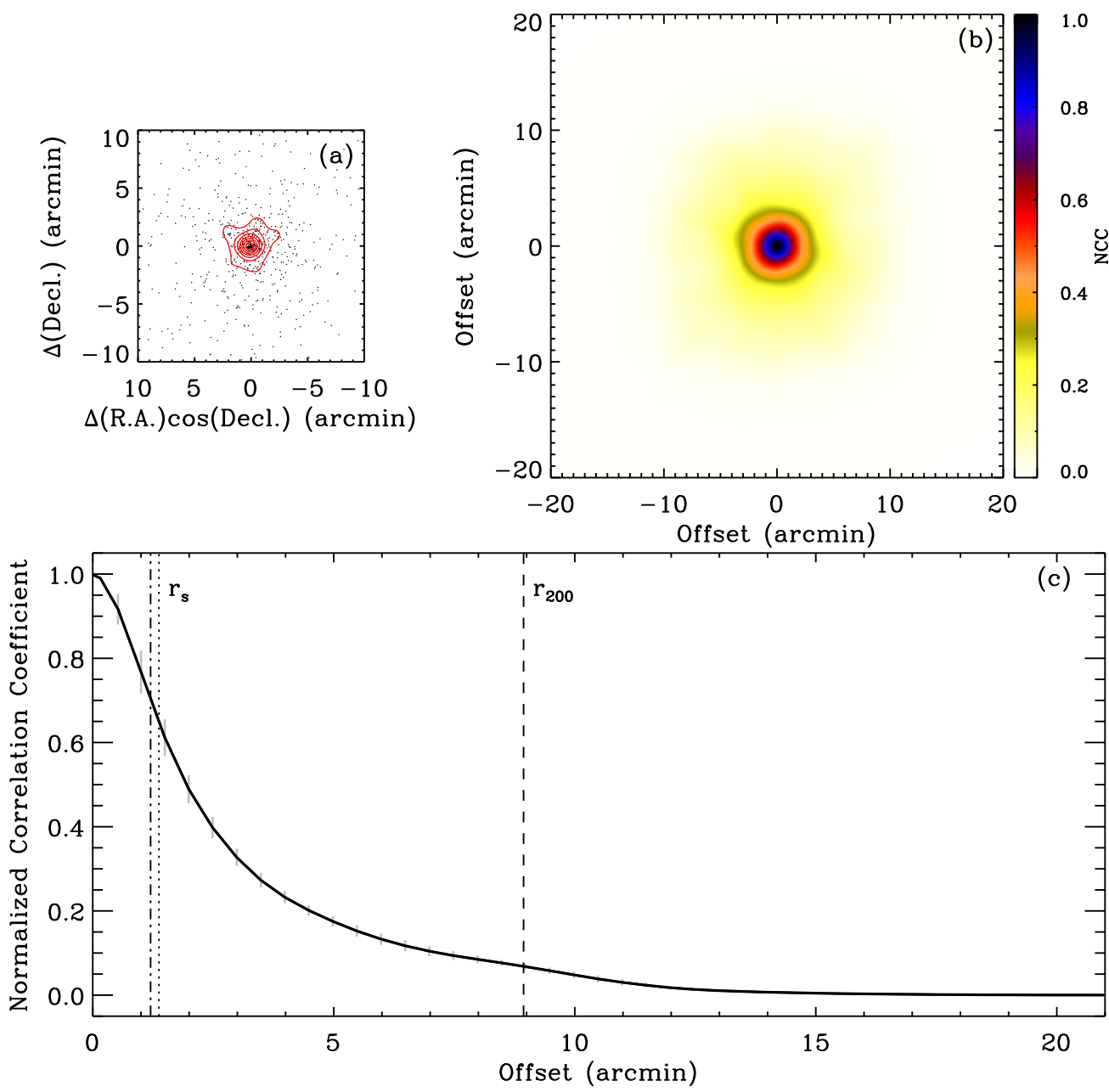

Figure 19. Normalized autocorrelation of the galaxy number density map for a simulated cluster. Top left: spatial distribution of galaxies (black dots) with galaxy number density contours (red). Top right: two-dimensional normalized autocorrelation map. Bottom: azimuthally averaged correlation signal as a function of offset. Vertical dot-dashed, dotted, and dashed lines indicate a smoothing scale FWHM $=1.2, r_{s}$, and $r_{200}$, respectively.

(A color version of this figure is available in the online journal.)

\section{APPENDIX \\ MATERIAL}

Here we provide two dimensional maps of the spectroscopic completeness for eight clusters in Figure 4 for A383: Figure 14 for A267 and A611, Figure 15 for A689 and A697, Figure 16 for A750 and A963, and Figure 17 for RX J1720.1+2638 and RX J2129.6+0005. We show the results of the autocorrelation for a galaxy number density map of a simulated cluster in Figures 18 (galaxy number density profile) and 19 (2D and 1D normalized autocorrelation signals).

\section{REFERENCES}

Ahn, C. P., Alexandroff, R., Allende Prieto, C., et al. 2014, ApJS, 211, 17 Arnouts, S., Cristiani, S., Moscardini, L., et al. 1999, MNRAS, 310, 540 Bahé, Y. M., McCarthy, I. G., \& King, L. J. 2012, MNRAS, 421, 1073 Becker, M. R., \& Kravtsov, A. V. 2011, ApJ, 740, 25

Broadhurst, T., Benítez, N., Coe, D., et al. 2005, ApJ, 621, 53 Carlberg, R. G., Yee, H. K. C., Ellingson, E., et al. 1996, ApJ, 462, 32

Clowe, D., Bradač, M., Gonzalez, A. H., et al. 2006, ApJL, 648, L109

Coe, D., Umetsu, K., Zitrin, A., et al. 2012, ApJ, 757, 22

Coupon, J., Broadhurst, T., \& Umetsu, K. 2013, ApJ, 772, 65

Csabai, I., Budavári, T., Connolly, A. J., et al. 2003, AJ, 125, 580

Diaferio, A. 1999, MNRAS, 309, 610

Diaferio, A., \& Geller, M. J. 1997, ApJ, 481, 633

Dodelson, S. 2004, PhRvD, 70, 023008
Drinkwater, M. J., Jurek, R. J., Blake, C., et al. 2010, MNRAS, 401, 1429 Duffy, A. R., Schaye, J., Kay, S. T., \& Dalla Vecchia, C. 2008, MNRAS, 390, L64

Fabricant, D., Fata, R., Roll, J., et al. 2005, PASP, 117, 1411

Geller, M. J., Dell'Antonio, I. P., Kurtz, M. J., et al. 2005, ApJL, 635, L125

Geller, M. J., Diaferio, A., Rines, K. J., \& Serra, A. L. 2013, ApJ, 764, 58

Geller, M. J., Hwang, H. S., Diaferio, A., et al. 2014a, ApJ, 783, 52

Geller, M. J., Hwang, H. S., Fabricant, D. G., et al. 2014b, ApJS, 213, 35

Geller, M. J., Kurtz, M. J., Dell'Antonio, I. P., Ramella, M., \& Fabricant, D. G. 2010, ApJ, 709, 832

Giles, P. A., Maughan, B. J., Birkinshaw, M., Worrall, D. M., \& Lancaster, K. 2012, MNRAS, 419, 503

Girardi, M., Boschin, W., \& Barrena, R. 2006, A\&A, 455, 45

Gonzalez, R. C., \& Woods, R. E. 2002, Digital Image Processing (2nd ed.; Upper Saddle River, NJ: Prentice-Hall)

Gruen, D., Bernstein, G. M., Lam, T. Y., \& Seitz, S. 2011, MNRAS, 416, 1392 Hoekstra, H. 2001, A\&A, 370, 743

Hoekstra, H. 2003, MNRAS, 339, 1155

Hoekstra, H. 2007, MNRAS, 379, 317

Hoekstra, H., Donahue, M., Conselice, C. J., McNamara, B. R., \& Voit, G. M. 2011a, ApJ, 726, 48

Hoekstra, H., Hartlap, J., Hilbert, S., \& van Uitert, E. 2011b, MNRAS, 412, 2095

Hu, W. 1999, ApJL, 522, L21

Huterer, D. 2010, GReGr, 42, 2177

Huterer, D., Kirkby, D., Bean, R., et al. 2013, arXiv:1309.5385

Ilbert, O., Arnouts, S., McCracken, H. J., et al. 2006, A\&A, 457, 841

Jaffé, Y. L., Poggianti, B. M., Verheijen, M. A. W., Deshev, B. Z., \& van Gorkom, J. H. 2013, MNRAS, 431, 2111

Johnston, D. E., Sheldon, E. S., Tasitsiomi, A., et al. 2007a, ApJ, 656, 27 
Johnston, D. E., Sheldon, E. S., Wechsler, R. H., et al. 2007b, arXiv:0709.1159 Kaiser, N. 1987, MNRAS, 227, 1

Kurtz, M. J., Geller, M. J., Utsumi, Y., et al. 2012, ApJ, 750, 168

Leauthaud, A., Finoguenov, A., Kneib, J.-P., et al. 2010, ApJ, 709, 97

Lemze, D., Postman, M., Genel, S., et al. 2013, ApJ, 776, 91

Mazzotta, P., Markevitch, M., Vikhlinin, A., et al. 2001, ApJ, 555, 205

Medezinski, E., Broadhurst, T., Umetsu, K., et al. 2010, MNRAS, 405, 257

Miyazaki, S., Komiyama, Y., Sekiguchi, M., et al. 2002, PASJ, 54, 833

Navarro, J. F., Frenk, C. S., \& White, S. D. M. 1997, ApJ, 490, 493

Newman, A. B., Treu, T., Ellis, R. S., et al. 2013, ApJ, 765, 24

Okabe, N., Takada, M., Umetsu, K., Futamase, T., \& Smith, G. P. 2010, PASJ, 62,811

Okabe, N., \& Umetsu, K. 2008, PASJ, 60, 345

Owers, M. S., Nulsen, P. E. J., \& Couch, W. J. 2011, ApJ, 741, 122
Prada, F., Klypin, A. A., Cuesta, A. J., Betancort-Rijo, J. E., \& Primack, J. 2012, MNRAS, 423, 3018

Regos, E., \& Geller, M. J. 1989, AJ, 98, 755

Rines, K., Geller, M. J., Diaferio, A., \& Kurtz, M. J. 2013, ApJ, 767, 15

Serra, A. L., \& Diaferio, A. 2013, ApJ, 768, 116

Serra, A. L., Diaferio, A., Murante, G., \& Borgani, S. 2011, MNRAS, 412, 800

Shan, H., Kneib, J.-P., Tao, C., et al. 2012, ApJ, 748, 56

Starikova, S., Jones, C., Forman, W. R., et al. 2014, ApJ, 786, 125

Umetsu, K., Medezinski, E., Nonino, M., et al. 2014, ApJ, 795, 163

Utsumi, Y., Miyazaki, S., Geller, M. J., et al. 2014, ApJ, 786, 93

Van Waerbeke, L., Benjamin, J., Erben, T., et al. 2013, MNRAS, 433, 3373

Zahid, J., Dima, G., Kudritzki, R., et al. 2014, ApJ, 791, 130

Zitrin, A., Broadhurst, T., Umetsu, K., et al. 2009, MNRAS, 396, 1985

Zitrin, A., Broadhurst, T., Umetsu, K., et al. 2010, MNRAS, 408, 1916 